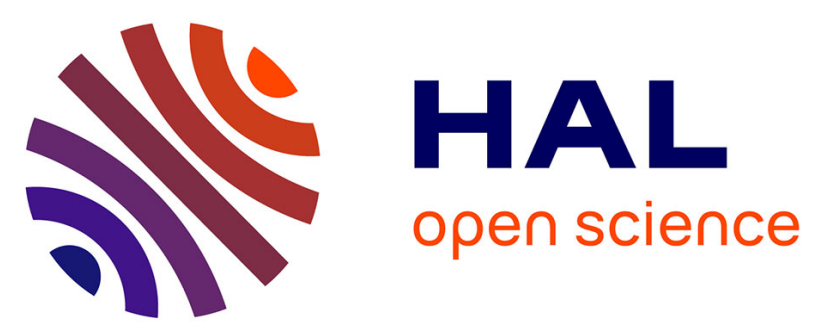

\title{
Full-Duplex and Backhaul-Constrained UAV-Enabled Networks using NOMA
}

Marie-Josépha Youssef, Joumana Farah, Charbel Abdel Nour, Catherine Douillard

\section{- To cite this version:}

Marie-Josépha Youssef, Joumana Farah, Charbel Abdel Nour, Catherine Douillard. Full-Duplex and Backhaul-Constrained UAV-Enabled Networks using NOMA. IEEE Transactions on Vehicular Technology, 2020, 69 (9), pp.9667-9681. 10.1109/TVT.2020.3001432 . hal-02901630

\section{HAL Id: hal-02901630 https://imt-atlantique.hal.science/hal-02901630}

Submitted on 17 Jul 2020

HAL is a multi-disciplinary open access archive for the deposit and dissemination of scientific research documents, whether they are published or not. The documents may come from teaching and research institutions in France or abroad, or from public or private research centers.
L'archive ouverte pluridisciplinaire HAL, est destinée au dépôt et à la diffusion de documents scientifiques de niveau recherche, publiés ou non, émanant des établissements d'enseignement et de recherche français ou étrangers, des laboratoires publics ou privés. 


\title{
Full-Duplex and Backhaul-Constrained UAV-Enabled Networks using NOMA
}

\author{
Marie-Josepha Youssef, Student Member, IEEE, Joumana Farah, Member, IEEE, Charbel Abdel Nour, Senior \\ Member, IEEE, and Catherine Douillard, Senior Member, IEEE.
}

\begin{abstract}
In this paper, a full-duplex unmanned aerial vehicle(UAV) base station is used to provide wireless communication to an area lacking a conventional terrestrial infrastructure, and its efficient deployment is investigated. More concretely, the UAV positioning and resource allocation problems are solved with the aim of minimizing the transmit power of the UAV, while serving users with their rate requirements and accounting for the backhaul limitation of the UAV. To this end, a complete solution for the optimal 3D position of the UAV, the bandwidth assignment and the transmit power distribution in the access and backhaul links is proposed that accounts for both the backhaul interference and the self-interference. When the UAV power budget is insufficient to fulfill rate requirements, nonorthogonal multiple access (NOMA) pairing is conducted to enhance system performance. Simulation results show that, when using the proposed approach, both the achieved sum rate and the percentage of satisfied users are significantly increased, when compared to a strategy that uses separate frequency bands in the access and backhaul links, as well as to a previously proposed method.
\end{abstract}

Index Terms-UAV-BS, wireless backhaul, in-band full-duplex, QoS requirements, NOMA.

\section{INTRODUCTION}

When used as flying base stations (BS), unmanned aerial vehicles (UAV) can help increase the throughput and the coverage of traditional communication systems thanks to their mobility, flexibility and low cost [1]. Moreover, they can help alleviate traffic congestion in hotspot areas and establish communication links in remote and disaster areas, where the communication infrastructure is either non-existent or damaged [2]. Therefore, their use in wireless communication systems has received a lot of attention in recent literature [3].

In [4], the authors investigated the optimal location of the UAV to minimize its transmit power. The optimal UAV altitude that maximizes its coverage region was evaluated in [5]. In [6], the authors built on the results of [5] and found the 3D location of the UAV that maximizes the coverage for users having different quality-of service (QoS) requirements.

Contrary to most terrestrial BSs, UAVs are connected to the core network through a wireless backhaul link [2], which was not accounted for in the works of [4]-[6]. That said, to reap the benefits promised by the use of UAV-BSs, this backhaul

Copyright (c) 2015 IEEE. Personal use of this material is permitted. However, permission to use this material for any other purposes must be obtained from the IEEE by sending a request to pubs-permissions@ieee.org

M. J. Youssef, C. Abdel Nour and C. Douillard are with IMT Atlantique, LabSTICC, UBL, F-29238 Brest, France, (e-mail: mariejosepha.youssef@imt-atlantique.fr; charbel.abdelnour@imt-atlantique.fr; catherine.douillard@imt-atlantique.fr). J. Farah is with the Department of Electricity and Electronics, Faculty of Engineering, Lebanese University, Roumieh, Lebanon (e-mail: joumanafarah@ul.edu.lb).

This work has been funded with support from the Lebanese University and IMT-Atlantique. link needs to be dynamically managed according to the traffic state of the network [3]. In fact, if not configured properly, the backhaul link introduces interference, thus limiting the throughput provided by the UAV in the access link. Moreover, in-band full-duplex (IBFD) communications were recently investigated to increase the spectral efficiency and reduce the latency of systems relying on a wireless backhaul [7]. When adopting an in-band wireless backhaul link, the same frequency band is used in the access and the backhaul links, thus optimizing the system spectral efficiency. Additionally, the deployment of in-band wireless backhauling is of great interest to cellular operators, since networks can be upgraded in a short time and a cost-effective way [8]. Moreover, IBFD allows the simultaneous transmission and reception of backhaul and access information in the same frequency band, at the expense of a self-interference (SI), induced by the transmitter on its own receiver. Nonetheless, SI cancellation schemes have progressed significantly [9], allowing an efficient application of IBFD for wireless backhauling.

The backhaul connectivity of UAV-enabled networks was discussed in a few recent work. The 3D placement problem of the UAV was investigated in [2] for the sake of maximizing the number of served users while considering a backhaul link with constant transmission rate. The authors in [10] introduced a heuristic algorithm that finds the number of needed UAVs as well as their 3D positions and accounts for the backhaul constraint. However, the authors did not elaborate on the way bandwidth assignment in the backhaul link is conducted. In [11], the authors proposed an algorithm to find the 3D position of the UAVs as well as the user and bandwidth allocation to maximize the logarithmic rates of users. Nevertheless, [11] assumed that access and backhaul transmissions take place on different and sufficiently spaced frequency bands to avoid SI.

In addition to UAV enabled networks, non-orthogonal multiple access (NOMA) has been recently proposed as a promising solution to enhance the performance of future communication networks [12]-[14]. In Power-Domain NOMA, multiple users are scheduled on the same time-frequency resource by multiplexing their signals in the power domain. At the receiver side, successive interference cancellation (SIC) is performed to retrieve the superimposed signals. By allowing multiple users to access the same resource, NOMA enhances spectral efficiency and user fairness.

Resource allocation for NOMA systems has been extensively studied with different performance measures. For example, the weighted sum rate of a full-duplex (FD) NOMA system was maximized in [15]; however the proposed method has exponential complexity. Maximizing system fairness was the target of [16], while minimizing the transmit power subject to rate requirements was targeted in [17] and [18]. [19] and 
[20] studied a distributed-antenna system (DAS) where the objective was to enhance the performance of a NOMA system characterized by a heterogeneous traffic. In the context of DAS also, [21] proposed several techniques to reduce the total transmit power in each cell.

The use of NOMA in UAV-enabled networks was given some attention in a few recent papers. In [22], the macro base station (MBS) performed backhaul transmission to dispatched UAVs using NOMA, with the UAVs cooperating to increase the data rates and reliability of served users. However, the study considered a half-duplex system, as the backhaul and access transmissions occurred in different timeslots. Moreover, NOMA was only considered for the transmission in the backhaul link. In [23], system performance of a NOMA UAV-enabled system was evaluated using stochastic geometry, and a reinforcement learning approach for the placement and movement of the UAV was proposed. The authors in [24] considered a NOMA-UAV system and maximized the minimum rate achieved with respect to the UAV altitude, beamwidth, power and bandwidth allocation. In [25], the sum rate of the users served by the UAV and the base station (BS) was maximized through the optimization of the user scheduling, the UAV trajectory and the precoding at the BS level. However, neither [23], [24] nor [25] considered the backhaul limitation of the UAV.

In this paper, we consider a scenario where the traditional wireless infrastructure is missing, e.g., in remote areas or due to a disaster or BS failure. An FD-UAV is dispatched to serve users having QoS requirements in that area, and an in-band wireless backhaul link is established between the UAV and an MBS to provide the needed backhaul capacity. To minimize the UAV transmit power, an optimization problem that finds the assignment of subbands in the backhaul link, the 3D position of the UAV as well as the power levels in the access and backhaul links is solved. Moreover, when the UAV power budget is not sufficient to guarantee user rate requirements, a NOMA pairing step is conducted to maximize the achievable rates. This is in constrast with our previous work in [26] where we assumed that the UAV power budget is sufficient to guarantee rate requirements.

The main contributions of this paper can be summarized as follows:

- We formulate and solve a feasibility test that checks if the user rate requirements can be simultaneously met with the UAV power budget.

- An optimization problem that minimizes the UAV transmit power is formulated. This problem takes into account the rate requirement per user, the backhaul constraint, and the transmit power budget constraints for the UAV and the MBS.

- A novel framework is introduced to find the best bandwidth assignment in the backhaul link, as well as the region in space in which the UAV can be deployed. Moreover, the number and the assignment of subbands in the backhaul link are optimized.

- After backhaul subband assignment, we determine the UAV position within the identified region and the power variables that lead to the minimum needed UAV power.
- When the UAV power budget cannot satisfy all users simultaneously, a NOMA pairing algorithm is proposed in order to maximize the achieved sum rate and number of satisfied users.

- To the best of our knowledge, this is the first work that proposes a complete solution for the joint problem of subband and power allocation in the access and the backhaul links, the UAV placement, as well as the NOMA pairing dedicated to enhance system performance.

The rest of this paper is organized as follows. In Sections II and III, the system model and the problem formulation are respectively described. Section IV introduces the foundations of our method while Section V details the proposed solution. In Section VI, the case where the UAV power budget is insufficient to guarantee the rate requirements is discussed. Finally, simulation results are presented in Section VII, before drawing the conclusions in Section VIII.

\section{SySTEM MODEL}

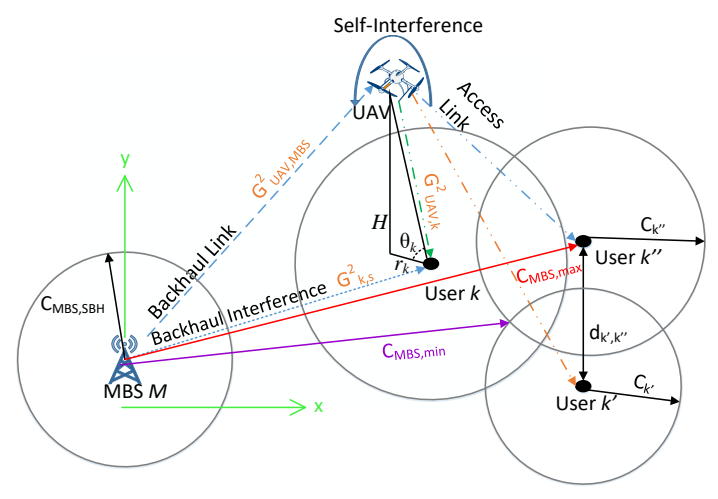

Fig. 1: System Model

The downlink UAV-enabled communication network consists of one IBFD-enabled UAV and $K$ randomly deployed users, as shown in Fig. 1. Note that the elements of Fig. 1 will be detailed as the paper progresses. The UAV is connected to the core network through an MBS $M$, located at the origin, via wireless in-band backhauling on the sub- $6 \mathrm{GHz}$ spectrum. The set of users is denoted by $\mathcal{K}$, with $R_{k}^{\text {req }}$ and $\left(x_{k}, y_{k}\right)$ indicating the rate requirement and the location of user $k \in \mathcal{K}$, respectively. Let the UAV position be denoted by $\boldsymbol{z}_{\boldsymbol{U} \boldsymbol{A} \boldsymbol{V}}=\left(x_{U A V}, y_{U A V}, H\right)$, where $\left(x_{U A V}, y_{U A V}\right)$ refers to the horizontal position of the UAV, while $H$ refers to its altitude. Clearly, the UAV should be positioned to serve as many users as possible, while being able to receive the necessary rate from the backhaul link.

Due to the IBFD wireless backhaul assumption, a user associated with the UAV suffers from the interference of the backhaul link occurring in the same frequency band. Moreover, the UAV suffers from SI.

\section{A. Path Loss Model}

The widely adopted air-to-ground (A2G) communication model [27] is considered between the UAV and the users, with 
two line-of-sight (LOS) and non-line-of-sight (NLOS) pathloss models. The probability of having a LOS communication link between the UAV and user $k$ is given by:

$$
P_{L O S}=\frac{1}{1+\alpha \exp \left(-\beta\left(\frac{180 \theta_{k}}{\pi}-\alpha\right)\right)},
$$

where $\theta_{k}=\arctan \left(\frac{H}{r_{k}}\right)$ is the elevation angle, $r_{k}=$ $\sqrt{\left(x_{k}-x_{U A V}\right)^{2}+\left(y_{k}-y_{U A V}\right)^{2}}$ is the horizontal distance between user $k$ and the UAV, whereas $\alpha$ and $\beta$ are constants determined by the environment (rural, urban, etc).

The average path-loss is taken as [6]:

$$
\begin{aligned}
L\left(H, r_{k}\right) & =\eta_{L} P_{L O S}+\eta_{N L} P_{N L O S}+20 \log \left(\frac{4 \pi f_{c} d_{U A V, k}}{c}\right) \\
& =\frac{A}{1+\alpha \exp \left(-\beta\left(\frac{180 \theta_{k}}{\pi}-\alpha\right)\right)}+20 \log \left(\frac{r_{k}}{\cos \left(\theta_{k}\right)}\right) \\
& +B
\end{aligned}
$$

where $P_{N L O S}=1-P_{L O S}, 20 \log \left(\frac{4 \pi f_{c} d_{U A V, k}}{c}\right)$ is the free space path loss, $f_{c}$ and $c$ being the carrier bandwidth and the speed of light respectively. Variables $\eta_{L}$ and $\eta_{N L}$ are the mean additional losses for LOS and NLOS links, respectively, whereas $d_{U A V, k}=\sqrt{\left(r_{k}^{2}+H^{2}\right)}$ is the $3 \mathrm{D}$ distance between the UAV and user $k$. In (2), $A=\eta_{L}-\eta_{N L}$ and $B=20 \log \left(\frac{4 \pi f_{c}}{c}\right)+\eta_{N L}$.

\section{B. Communication Model}

We consider the sub-6 GHz spectrum where the system bandwidth $B W$ is equally partitioned into a set $\mathcal{S}$ of $S$ subbands, leading to a subband bandwidth of $B_{c}=B W / S$. In this study, it is assumed that $K=S$ and that each user $k$ is assigned a unique subband $s$ in the access link. Let $G_{k, s}$ be the channel gain between the MBS and each user $k$ over subband $s$, consisting of both small-scale and large-scale fading. The rate achieved by user $k$ over $s$ is given by [10]:

$$
R_{k, s}=B_{c} \log _{2}\left(1+\frac{P_{U A V, k, s} G_{U A V, k}^{2}}{N_{0} B_{c}+b_{s} P_{M B S, s} G_{k, s}^{2}}\right),
$$

where $P_{U A V, k, s}$ is the transmit power allocated by the UAV to user $k$ on subband $s$, while $P_{M B S, s}$ is the transmit power of the MBS on $s$. The noise power spectral density is $N_{0}$ and $G_{U A V, k}^{2}=10^{-L\left(H, r_{k}\right) / 10}$ is the channel gain between the UAV and user $k$. The binary decision variable $b_{s} \in\{0,1\}$ is equal to 1 if subband $s$ is used in the backhaul link, and 0 otherwise. When $b_{s}=1, P_{M B S, s} G_{k, s}^{2}$ denotes the backhaul interference (BI) suffered by user $k$.

The channel between the UAV and the MBS also follows the A2G path-loss model. When $b_{s}=1$, the rate achieved by the UAV over $s$ is given by:

$$
R_{U A V, s}=B_{c} \log _{2}\left(1+\frac{P_{M B S, s} G_{U A V, M B S}^{2}}{N_{0} B_{c}+C_{S I} \sum_{k \in \mathcal{K}} P_{U A V, k, s}}\right),
$$

where $G_{U A V, M B S}^{2}$ is the channel gain between the UAV and the MBS. The residual SI experienced at the UAV on subband $s$ is denoted by $C_{S I} \sum_{k} P_{U A V, k, s}$, with $1 / C_{S I}$ being the SI cancellation factor.

\section{PRoblem Formulation}

UAV communications being energy-limited, the main purpose of this study is to minimize the transmit power of the UAV while meeting the rate requirements of all users:

$$
\begin{aligned}
& \min _{\boldsymbol{P}_{\boldsymbol{z}_{\boldsymbol{U} \boldsymbol{U} \boldsymbol{\boldsymbol { V }}}, \boldsymbol{P}_{\boldsymbol{M} \boldsymbol{B} \boldsymbol{S}}, \boldsymbol{a}, \boldsymbol{b}}} \sum_{k \in \mathcal{K}} \sum_{s \in \mathcal{S}} a_{k, s} P_{U A V, k, s} \\
& \text { such that } \sum_{s \in \mathcal{S}} a_{k, s} R_{k, s} \geq R_{k}^{\mathrm{req}}, \forall k \in \mathcal{K} \text {, } \\
& \sum_{k \in \mathcal{K}} \sum_{s \in \mathcal{S}} a_{k, s} R_{k, s} \leq \sum_{s \in \mathcal{S}} b_{s} R_{U A V, s} \\
& \sum_{k \in \mathcal{K}} \sum_{s \in \mathcal{S}} P_{U A V, k, s} \leq P_{U A V}^{\max }, \\
& \sum_{s \in \mathcal{S}} b_{s} P_{M B S, s} \leq P_{M B S}^{\max }, \\
& H_{\text {min }} \leq H \leq H_{\max }, \\
& a_{k, s}, b_{s} \in\{0,1\} \text {. }
\end{aligned}
$$

In (5), $a_{k, s}$ is a binary variable that is equal to 1 if user $k$ is scheduled on subband $s$ in the access link and 0 otherwise. (5a) is the rate constraint for each user, while $(5 b)$ is the backhaul data rate constraint which ensures that the total rate delivered by the UAV to users does not exceed its backhaul capacity. In (5c) and (5d), $P_{U A V}^{\max }$ and $P_{M B S}^{\max }$ are the transmit power budgets of the UAV and the MBS respectively. (5e) is the altitude constraint for the UAV.

Problem (5) consists of three subproblems: the UAV placement problem, as well as the problems of bandwidth and power allocation in the access and backhaul links. One can see that, at the optimum, constraint (5a) is satisfied with equality for all users. Hence, the left hand side of (5b) can be substituted with $\sum_{k} R_{k}^{\text {req }}$. Nonetheless, the resulting formulated optimization problem is mixed-integer and multivariate. To solve it, we propose a multi-step algorithm that targets the three subproblems.

Once the subband assignment in the access link, $\boldsymbol{a}$, is known, solving (5) resorts to finding the backhaul subband assignment $\boldsymbol{b}$, the UAV position $\boldsymbol{z}_{\boldsymbol{U} \boldsymbol{A} \boldsymbol{V}}$ and the power variables $\boldsymbol{P}_{U A V}$ and $\boldsymbol{P}_{M B S}$ minimizing the UAV transmit power. Therefore, the proposed solution proceeds as follows:

1) Perform the subband assignment in the access link.

2) Solve a feasibility test to check whether $P_{U A V}^{\max }$ can accommodate the rate requirements without considering the BI.

3) Decide on the assignment of backhaul subbands. To do so:

a) Determine the minimum number of required backhaul subbands. This initial value does not take into account the impact of the resulting BI at the user side.

b) Retain the backhaul subband assignment minimizing the needed UAV power and accounting for the resulting BI at the user side.

4) Find $z_{U A V}, P_{U A V}$, and $\boldsymbol{P}_{M B S}$

5) If needed, perform a NOMA pairing step to further improve achieved user data rates. 


\section{PRELIMINARIES}

First, the subband assignment in the access link is discussed before detailing the initialization steps.

\section{A. Subband Assignment in the Access Link}

As per Eq. (3), to reach the users required rates while achieving the objective of problem (5), the subband assignment in the access link should take into account:

- maximizing the average channel gain between the UAV and its users, i.e., maximizing $G_{U A V, k}, \forall k$ in $\mathcal{K}$,

- minimizing the average BI experienced by the users, i.e., minimizing the average channel gain between the MBS and each user, $G_{k, s}, \forall k$ in $\mathcal{K}$.

Since the adopted A2G channel model [27] does not consider frequency selectivity, $G_{U A V, k}$ is constant $\forall s \in \mathcal{S}$ and hence does not affect the optimization of (5) from the perspective of subband assignment in the access link. Consequently, achieving the objective of problem (5) comes down to minimizing the average experienced BI by minimizing the average channel gain between the MBS and each user on its allocated subband. Therefore, we propose to solve the following optimization problem:

$$
\begin{gathered}
\min _{\boldsymbol{a}} \sum_{k \in \mathcal{K}} \sum_{s \in \mathcal{S}} a_{k, s} G_{k, s} \\
\text { such that } \sum_{s \in \mathcal{S}} a_{k, s}=1, \forall k \in \mathcal{K} .
\end{gathered}
$$

Problem (6) aims at finding the assignment $\boldsymbol{a}$ that minimizes the average channel gain between users and the MBS, hence the average BI. Constraint (6a) restricts each user to be assigned one subband only. Being an assignment problem, (6) can be efficiently solved using the Hungarian method [28].

Next, a method to find the 2D UAV deployment region guaranteeing constraints (5a) and (5b) is described.

\section{B. Finding the coverage region for each user and for the MBS}

Once the subband allocation in the access link is known, for each value of $P_{U A V, k, s}$ and $P_{M B S, s}$, the rate requirement in (5a) can be translated into a requirement on the maximum tolerable path-loss. To guarantee $R_{k, s} \geq R_{k}^{\text {req }}$, from (3), the channel gain between the UAV and user $k, G_{U A V, k}^{2}$ must satisfy:

$$
G_{U A V, k}^{2} \geq\left(2^{\frac{R_{k}^{\mathrm{req}}}{B_{c}}}-1\right) \frac{N_{0} B_{c}+b_{s} P_{M B S, s} G_{k, s}^{2}}{P_{U A V, k, s}} .
$$

By noting that $G_{U A V, k}^{2}=10^{-L\left(H, r_{k}\right) / 10}$, the path-loss experienced by user $k, L\left(H, r_{k}\right)$, should satisfy:

$$
\begin{aligned}
& L\left(H, r_{k}\right) \leq L_{k}^{\mathrm{th}}= \\
& -10 \log _{10}\left\{\left(2^{\frac{R_{k}^{\mathrm{req}}}{B_{c}}}-1\right) \frac{N_{0} B_{c}+b_{s} P_{M B S, s} G_{k, s}^{2}}{P_{U A V, k, s}}\right\},
\end{aligned}
$$

where $L_{k}^{\text {th }}$ is the maximum tolerable path-loss by user $k$ to reach $R_{k}^{\text {req }}$. In other words, user $k$ meets its rate requirement if its experienced path-loss $L\left(H, r_{k}\right)$ is less than or equal to the maximum tolerable path-loss $L_{k}^{\text {th }}$. For a given UAV altitude
$H$, let $C_{k}(H)$ be the maximum 2D distance between the UAV and user $k$ guaranteeing $R_{k}^{\text {req }}$, i.e., $C_{k}(H)=\left.r\right|_{L_{(H, r)}=L_{k}^{\text {th }}}$.

From Eq. (2), for a given value of $H$, if the UAV is located at a distance $C_{k}(H)$ from user $k$, the latter experiences a constant path loss $L_{k}^{\text {th }}$. Moreover, if the UAV is located at a distance $r \leq C_{k}(H)$ from user $k$, the latter experiences a path loss $L(H, r) \leq L_{k}^{\text {th }}$. Hence, from the perspective of user $k$, its rate requirement is met if the UAV is positioned inside the circular disk having user $k$ as center and $C_{k}(H)$ as radius. It was shown in [5] that, for a fixed maximum path-loss, the coverage radius of the UAV as a function of $H$ has one optimum point only which corresponds to the largest coverage radius. In this work, we propose to make use of the inverse relation which equally holds, since the channel between the UAV and the users is assumed symmetrical. In other words, from the perspective of user $k$, the coverage radius $C_{k}(H)$ has one maximum value denoted as $C_{k}$. Hence, finding the maximum value of $C_{k}(H)$ consists in finding the optimal altitude that results in the maximum coverage radius $C_{k}$. It was also shown in [5], [6] that finding the optimal altitude consists in solving $\frac{\partial \theta}{\partial H}=0$ and that $\theta_{\text {opt }}$ which achieves the optimum $C_{k}$ depends only on the considered environments. For the suburban, urban and dense-urban environments, $\theta_{\text {opt }}=20.34^{\circ}, 42.44^{\circ}, 54.62^{\circ}$ respectively.

Having $\theta_{\mathrm{opt}}, C_{k}$ can be found using (2) as follows:

$$
C_{k}=\cos \left(\theta_{\text {opt }}\right) \times 10^{L_{k}^{\text {th }} / 20} \times 10^{-D / 20}=E \times 10^{L_{k}^{\text {th }} / 20},
$$

where $D=\frac{A}{1+\alpha \exp \left(-\beta\left(180 \theta_{\mathrm{opt}} / \pi-\alpha\right)\right)}+B$ and $E=\cos \left(\theta_{\mathrm{opt}}\right) \times$ $10^{-D / 20}$.

We propose to associate every user $k$ with a 2D coverage region of radius $C_{k}$ (Cf. Fig. 1) that guarantees its rate requirement, given the power values in the access and backhaul links. Let $D_{k}$ be the disk centered at user $k$ and of radius $C_{k}$. It should be noted that $C_{k}$ will only be used to find the acceptable 2D deployment region for the UAV where all users can be served simultaneously.

Similarly, when considering the backhaul link, the UAV should be positioned in a region where it is able to receive the required backhaul rate from the MBS. Let $S_{B H}$ be the value of the total number of backhaul subbands. To meet the required backhaul rate, from (4), the path-loss between the UAV and the MBS should satisfy:

$$
\begin{aligned}
L\left(H, r_{M B S}\right) \leq & L_{S_{B H}}^{\text {h }}=-10 \log _{10}\left\{\left(2^{R_{U A V, s} / B_{c}}-1\right) \times\right. \\
& \left.\frac{N_{0} B_{c}+C_{S I} \sum_{k \in \mathcal{K}} P_{U A V, k, s}}{P_{M B S, s}}\right\} .
\end{aligned}
$$

Note that (10) depends on the value of the total number of backhaul subbands, $S_{B H}$, through both $R_{U A V, s}$ and $P_{M B S, s}$, the rate and the power allocated by the MBS to each subband used in the backhaul link, respectively.

As in the users case, the maximum coverage region of the MBS satisfying the backhaul path-loss requirement is found using (9) after replacing $L_{k}^{\text {th }}$ with $L_{S_{B H}}^{\text {th }}$. Being a function of the number of backhaul subbands, the maximum coverage radius of the MBS is denoted by $C_{M B S, S_{B H}}$. Let $D_{M B S, S_{B H}}$ 
be the disk centered at the MBS with $C_{M B S, S_{B H}}$ as radius.

Having found the $2 \mathrm{D}$ region guaranteeing (5a) for each user and (5b) in the backhaul link, a feasibility test that checks if the available UAV power is sufficient for guaranteeing the intersection of the different coverage regions is performed next. If positive, the number of backhaul subbands minimizing the needed UAV power when accounting for the BI is then found. In the opposite case, problem (5) is still solved while initially relaxing constraint $(5 \mathrm{c})$. After finding the UAV position, a subsequent NOMA pairing step is then performed to maximize the achieved rates while enforcing back (5c).

\section{Feasibility Test}

The feasibility test that checks if $P_{U A V}^{\max }$ is sufficient to guarantee (5a) is done when considering that none of the subbands is used in the backhaul link, i.e., $b_{s}=0, \forall s \in \mathcal{S}$. If the test is negative without considering any $\mathrm{BI}$, the system is infeasible under any combination of used backhaul subbands because of the added interference. A negative feasibility test means that the current rate requirements cannot be met simultaneously with the available UAV power.

Let $s_{k}$ be the subband assigned to user $k$. When replacing $L_{k}^{\text {th }}$ in (9) by its expression from (8), and by noting that $10^{L_{k}^{\text {th } / 20}}=\frac{\sqrt{P_{U A V, k, s}}}{\sqrt{\left(2^{R_{k}^{\text {req }} / B_{c}}-1\right) N_{0} B_{c}}}$ in the absence of BI, $C_{k}$ can be formulated as:

$$
C_{k}=\frac{E \times \sqrt{P_{U A V, k, s_{k}}}}{\sqrt{\left(2^{R_{k}^{\mathrm{req}} / B_{c}}-1\right) N_{0} B_{c}}} .
$$

For the problem to be feasible, a set of access power values $\boldsymbol{P}_{\boldsymbol{U} \boldsymbol{A} \boldsymbol{V}}$, that guarantees the intersection of the different user coverage regions and satisfies the UAV power budget, i.e., constraint $(5 \mathrm{c})$, must be found. Therefore, the following optimization problem is formulated:

$$
\begin{gathered}
\min _{\boldsymbol{P}_{\boldsymbol{U} \boldsymbol{A} \boldsymbol{V}}} \sum_{k \in \mathcal{K}} P_{U A V, k, s_{k}} \\
\text { such that } \bigcap_{k \in \mathcal{K}} D_{k} \neq \emptyset .
\end{gathered}
$$

Constraint (12a) ensures that the coverage disk of each user intersects with the coverage disks of all other users. In other words, (12a) ensures the existence of a space region where, if positioned, the UAV can simultaneously guarantee the rate requirements of all users. Note that the MBS coverage region is not taken into account in (12) since the feasibility test is performed while considering that $b_{s}=0, \forall s \in \mathcal{S}$.

To account for (12a), Helly's theorem [29] can be used: given $M$ convex sets of dimension $n$, if the intersection of every $(n+1)$ combination of the $M$ sets is non empty, so is the intersection of all $M$ sets. Although the solution using Helly's theorem is precise, the execution time is in the order of $\mathcal{O}\left(M^{3}\right)$. Therefore, a less computationally demanding solution is presented next.

First, we note that the joint intersection of all coverage disks is non empty if we can find at least one point that belongs to all disks. Therefore, (12) is reformulated as follows:

$$
\begin{gathered}
\underset{\boldsymbol{P}_{\boldsymbol{U} \boldsymbol{A} \boldsymbol{V}}, \boldsymbol{o}}{ } \sum_{k \in \mathcal{K}} P_{U A V, k, s_{k}} \\
\text { such that } d_{k, \boldsymbol{o}} \leq C_{k}, \forall k \in \mathcal{K},
\end{gathered}
$$

where $\boldsymbol{o}$ is the intersection point in 2D space and $C_{k}$, the coverage radius of user $k$, is expressed in terms of $P_{U A V, k, s_{k}}$ using (11). Constraint (13a) ensures that $\boldsymbol{o}$ belongs to all $K$ disks by enforcing the distance between each user $k$ and $\boldsymbol{o}$, $d_{k, \boldsymbol{o}}$, to be less than the circle radius of user $k$. Hence, (13a) guarantees the intersection of all $K$ disks.

A solution for problem (13) can always be found, since the UAV power constraint is not enforced at this stage. Moreover, (13) is a convex optimization problem since it involves a linear objective function and convex inequality constraints. By writing the Lagrangian and solving the Karush-Kuhn-Tucker (KKT) conditions, the power values in the access link, $\boldsymbol{P}_{U \boldsymbol{A} \boldsymbol{V}}^{*}$, and the 2D coordinates of the intersection point $\boldsymbol{o},\left(x_{\boldsymbol{o}}, y_{\boldsymbol{o}}\right)$, can be found according to:

$$
\begin{gathered}
x_{\boldsymbol{o}}=\frac{\sum_{k} \tau_{k} x_{k}}{\sum_{k} \tau_{k}}, \\
y_{\boldsymbol{o}}=\frac{\sum_{k} \tau_{k} y_{k}}{\sum_{k} \tau_{k}}, \\
P_{U A V, k, s_{k}}^{*}=\tau_{k}\left[\left(x_{k}-x_{\boldsymbol{o}}\right)^{2}+\left(y_{k}-y_{\boldsymbol{o}}\right)^{2}\right],
\end{gathered}
$$

where $\tau_{k}=\left(2^{R_{k}^{\mathrm{req}} / B_{c}}-1\right) N_{0} B_{c} / E^{2}$.

If $\boldsymbol{P}_{U \boldsymbol{A} \boldsymbol{V}}^{*}$ satisfies the UAV power budget, problem (5) is feasible. In the opposite case, problem (5) is still solved according to the technique detailed in the next Section. However, constraint (5c) is relaxed. Additional steps are then performed to re-enforce the UAV power budget constraint while enhancing system performance. These additional steps are detailed in Section VI.

\section{Initialization of the Access Power Values}

As a result of solving (13), when (5) is feasible, the power values $\boldsymbol{P}_{\boldsymbol{U} \boldsymbol{A} \boldsymbol{V}}^{*}$ result in an intersection region of all coverage disks that is just a point in the 2D space. Indeed, in order to achieve the objective of problem (13), the smallest power values satisfying the constraint in (13a) are found. Therefore, for at least two coverage disks, the intersection is just a point.

To maximize the intersection region, the initial power values must be increased as much as possible so that the smallest pairwise intersection is maximized. Indeed, it is clear from (8) that an increase of $P_{U A V, k, s}$ results in an increase of $L_{k}^{\text {th }}$ and therefore in $C_{k}$. Put differently, for the two disks with the smallest intersection, their radii, hence the associated power values, are recomputed to increase the intersection. Therefore, the following optimization problem is solved:

$$
\max _{\boldsymbol{P}_{\boldsymbol{U}} \boldsymbol{A V} \boldsymbol{V}} \min _{k, k^{\prime} \in \mathcal{K}}\left\{\left(C_{k}+C_{k^{\prime}}\right)-d_{k, k^{\prime}}\right\}
$$


such that $P_{U A V, k, s_{k}} \geq P_{U A V, k, s_{k}}^{*}, \forall k \in \mathcal{K}$,

$$
\sum_{k \in \mathcal{K}} P_{U A V, k, s_{k}}=P_{U A V}^{\max } .
$$

In (17), $d_{k, k^{\prime}}$ is the 2D distance between users $k$ and $k^{\prime}$ (Cf. Fig. 1). Constraint (17a) ensures the updated power variables are at least equal to the ones found by the solution of (13), therefore preserving the intersection point found by (13).

Problem (17) is convex since it involves the minimization of the maximum of multiple convex functions. Hence, it can be solved efficiently to find the initial power values $\boldsymbol{P}_{U A V}^{i}$. When the feasibility test (13) is negative, the minimum required power in the access link, computed by (13), exceeds the UAV power budget. In this case, the values of $\boldsymbol{P}_{U A V}^{i}$ are set to the same values of $\boldsymbol{P}_{U A V}^{*}$ found by the solution of problem (13).

\section{E. MBS Coverage Radius}

In this section, the maximum MBS coverage radius for each potential number of subbands $S_{B H}$ is found.

To avoid the backhaul subband assignment step at this stage of the solution, for each potential number of subbands $S_{B H}$, equal power and rate repartition in the backhaul link are assumed. Note that this assumption is done in order to initialize the MBS coverage radius for each value of $S_{B H}$, and will be relaxed at later stages of the algorithm. With this assumption, the following observation is made.

Proposition 1. If $P_{M B S, s}$ and $R_{U A V, s}$ are constant $\forall s \in \mathcal{S}$, the path-loss threshold $L_{S_{B H}}^{\text {th }}$ in (10) decreases (i.e. becomes more strict) when the power in the access link increases.

Following Proposition 1, the minimum value of the required path-loss $L_{S_{B H}}^{\text {th }}$ is achieved when the access power value is the largest. Therefore, to compute $L_{S_{B H}}^{\text {th }}$, the highest value of $P_{U A V, k, s}^{i}$, found from (17), is used:

$$
\begin{aligned}
L_{S_{B H}}^{\mathrm{th}}= & -10 \log _{10}\left\{\left(2^{\frac{R^{\mathrm{req}}}{S_{B H} \times B_{c}}}-1\right) \times\right. \\
& \left.\frac{N_{0} B_{c}+C_{S I} \times \max _{s}\left(\sum_{k} P_{U A V, k, s}^{i}\right)}{P_{M B S}^{\max } / S_{B H}}\right\},
\end{aligned}
$$

where $R^{\text {req }}=\sum_{k} R_{k}^{\text {req }}$.

By considering this worst case situation, the strictest required path-loss from (10) is accounted for. Then, the MBS coverage radius for $S_{B H}$ backhaul subbands, $C_{M B S, S_{B H}}$, is found from (9) after replacing $L_{k}^{\text {th }}$ with $L_{S_{B H}}^{\text {th }}$. With $L_{S_{B H}}^{\text {th }}$ being the minimum value of the required path-loss, the computed $C_{M B S, S_{B H}}$ is also the smallest achievable value of the MBS coverage radius. Hence, $C_{M B S, S_{B H}}$ is guaranteed under any assignment of backhaul subbands.

Proposition 2. As shown in Appendix A, $C_{M B S, S_{B H}}$ is an increasing function of $S_{B H}$.

With the increase of $S_{B H}$ and $L_{S_{B H}}^{\text {th }}, C_{M B S, S_{B H}}$ increases to the point where the MBS coverage region encompasses the coverage regions of all users, when using the whole MBS power budget. However, an optimization problem that aims at minimizing the UAV transmit power is expected to position the UAV at a position that is close to the MBS and users at once. In other words, the UAV location should not be very far from the MBS since it would logically require more backhaul power to reach its backhaul rate requirement. This translates into more BI at the user side, which in its turn increases the needed UAV power to guarantee the access rate requirements. Hence, the following observation is made.

Proposition 3. The maximum needed backhaul coverage radius, $C_{M B S, \max }$ (Cf. Fig. 1), is given by the distance between the MBS and the user farthest from it.

Consequently, when $C_{M B S, S_{B H}}$, is larger than the maximum needed $C_{M B S, \max }$, it is replaced by $C_{M B S, \max }$.

When finding the MBS coverage radius in this way, the needed MBS power is ensured to respect the MBS power budget. In fact, for small values of $S_{B H}$ resulting in $C_{M B S, S_{B H}}<$ $C_{M B S, \max }$, the needed power is the whole MBS power budget divided equally between the $S_{B H}$ subbands. However, when $S_{B H}$ increases and results in substituting $C_{M B S, S_{B H}}$ with $C_{M B S, \max }$, the needed backhaul power is reduced with respect to the budget $P_{M B S}^{\max }$. Therefore, the needed MBS power, $P_{M B S}^{\text {needed }}$, always satisfies the MBS power budget, i.e., $P_{M B S}^{\text {needed }} \leq P_{M B S}^{\max }$.

\section{Proposed Solution}

\section{A. Finding the minimum number of required backhaul sub- bands}

To ensure that the UAV can provide the access rate to its users while meeting its backhaul rate requirement, it must be positioned in the intersection of the coverage region of all users and the coverage region of the MBS. The latter depends on $S_{B H}$. When $S_{B H}$ is small, the required data rate per subband, $R_{U A V, s}$, is naturally large. This causes the maximum tolerable path-loss $L_{S_{B H}}^{\text {th }}$, given in (10), to be small, resulting in a narrow MBS coverage radius. On the other hand, if all subbands are used in the backhaul link, the MBS coverage radius becomes large. However, for some users with high rate requirements, the added BI shrinks their coverage regions. This could result in an impossibility to serve all users simultaneously. Therefore, the number and the choice of backhaul subbands should be optimized.

To find the number of backhaul subbands that results in the minimum needed UAV power, for each potential value of $S_{B H}$, i.e., for $S_{B H}=\{1, \ldots, S\}$, the corresponding backhaul subbands must be chosen, and the needed UAV power must be found. However, to reduce the number of backhaul subbands values to be tested, the following observation is made.

Proposition 4. The largest simultaneous coverage region for the users, $D_{\mathrm{int}}^{\max }$, is achieved when none of the subbands is used in the backhaul link, i.e., when $b_{s}=0, \forall s \in \mathcal{S}$.

Proof. Let $D_{\text {int }}$ be the simultaneous coverage region when a subband $s$ is used in the backhaul link and let $k$ be the user scheduled on $s$. According to (8), $L_{k}^{\text {th }}$ decreases when the BI increases. Hence, the coverage region of $k$ becomes smaller when its allocated subband is used in the backhaul link, in comparison to the opposite case. Therefore, the simultaneous 
coverage region $D_{\text {int }}$ is smaller than $D_{\text {int }}^{\max }$, when one or more subbands are used in the backhaul link.

Following Proposition 4, any MBS coverage region that does not intersect with $D_{\text {int }}^{\max }$ does not intersect with the simultaneous user coverage region that accounts for BI. Let $\left(x_{0}, y_{0}\right) \in D_{\text {int }}^{\max }$ be the closest point in $2 \mathrm{D}$ space to the MBS, i.e., $\left(x_{0}, y_{0}\right)=\underset{(x, y) \in D_{\text {int }}^{\max }}{\operatorname{argmin}} \sqrt{x^{2}+y^{2}}$. In order to have an intersection with $D_{\text {int }}^{\max }$, the MBS coverage radius must satisfy:

$$
C_{M B S, S_{B H}} \geq C_{M B S, \min }=\sqrt{x_{0}^{2}+y_{0}^{2}} .
$$

The minimum value of the number of backhaul subbands, $S_{B H \text {,min }}$, satisfies condition (19) and is found using bisection search.

Having found $S_{B H \text {,min }}$ and the total backhaul power needed for every potential value of $S_{B H}$, we now turn our attention to the choice of these subbands.

\section{B. Deciding on the number and choice of backhaul subbands}

When finding the required MBS path-loss and maximum MBS coverage radius in section IV-E, the maximum power value in the access link was taken without considering BI. Hence, the value of $S_{B H \text {,min }}$ was found while considering maximum SI but in the absence of BI. When accounting for the BI, the coverage regions of users decrease in size. Therefore, it is not guaranteed that all subbands numbers in the set $\mathcal{S}_{B H}^{\text {poss }}=\left\{S_{B H, \min }, \ldots, S\right\}$ ensure that the coverage regions of users intersect with the one of the MBS on one hand, and with each other on the other, while respecting the UAV power budget. Hence, we introduce, in this subsection, an algorithm to find the value of $S_{B H}$ that results in the best performance.

Starting from the case where all subbands are used in the backhaul link, i.e., $S_{B H}=S$, problem (20) is solved to find the needed backhaul power per subband:

$$
\begin{gathered}
\min _{\boldsymbol{P}_{M B S}} \sum_{s \in \mathcal{S}_{S_{B H}}} P_{M B S, s}^{S_{B H}} \\
\text { such that } \sum_{s \in \mathcal{S}_{S_{B H}}} R_{U A V, s}=R^{\mathrm{req}} .
\end{gathered}
$$

In (20a), $\mathcal{S}_{S_{B H}}$ is the set of subbands used in the backhaul link when the total number of backhaul subbands is $S_{B H}$ and $R_{U A V, s}$ is given by Eq. (4) while considering the initial access power values found in section IV-D. In addition, at this point, the UAV is assumed to be positioned at the maximum tolerable distance from the MBS. In other words, the distance between the UAV and the MBS is equal to the MBS coverage radius $C_{M B S, S_{B H}}$.

Finding the Lagrangian of (20) and setting it to zero yields:

$$
P_{M B S, s}^{S_{B H}}=\frac{\lambda}{\log (2)}-\frac{N_{0} B_{c}+C_{S I} \sum_{k} P_{U A V, k, s}^{i}}{10^{-L_{S_{B H}}^{\mathrm{th}} / 10}},
$$

where $\lambda$ is the Lagrange multiplier given by:

$$
\lambda=2^{\frac{1}{S_{B H}}\left(\frac{R^{\mathrm{req}}}{B_{c}}-\sum_{s \in \mathcal{S}_{S_{B H}}} \log _{2}\left(\frac{10^{-L_{S_{B H}}^{\mathrm{th}} / 10}}{\log (2)\left(N_{0} B_{c}+C_{S I} \sum_{k} P_{U A V, k, s}^{i}\right)}\right)\right) .}
$$

The MBS power values resulting from (21) are found while using the access power values $\boldsymbol{P}_{\boldsymbol{U} \boldsymbol{A} \boldsymbol{V}}$ which do not account for the BI. Therefore, at this stage, the access power values accounting for the BI are re-calculated in a similar manner to that in section IV-C: The maximum coverage radius for user $k$ is given by (9). However, the path-loss thresholds must be modified to account for the BI. For this purpose, in this section, equation (11) is substituted with the following expression:

$$
C_{k, S_{B H}}=\frac{E \times \sqrt{P_{U A V, k, s_{k}}}}{\sqrt{\left(2^{R_{k}^{\mathrm{req}} / B_{c}}-1\right)\left(N_{0} B_{c}+G_{k, s_{k}}^{2} P_{M B S, s_{k}}^{S_{B H}}\right)}} .
$$

To find the access power needed for $S_{B H}$ backhaul subbands, the following convex optimization problem is solved:

$$
\begin{gathered}
\min _{\boldsymbol{P}_{\boldsymbol{U} \boldsymbol{A} \boldsymbol{V}}^{\boldsymbol{S}_{B H}, \boldsymbol{o}}} \sum_{k \in \mathcal{K}} P_{U A V, k, s_{k}}^{S_{B H}} \\
\text { such that } d_{k, \boldsymbol{o}} \leq C_{k, S_{B H}}, \forall k \in \mathcal{K}, \\
d_{M B S, \boldsymbol{o}} \leq C_{M B S, S_{B H}} .
\end{gathered}
$$

(24) is a generalization of (13) that accounts for the BI. Constraint (24b) ensures the joint intersection of the MBS coverage region and the coverage region of each user.

Being mutually dependent, problems (20) and (24) are repeatedly solved until convergence, i.e., until the change in the values of $\boldsymbol{P}_{M B \boldsymbol{S}}^{\boldsymbol{S}_{B H}}$ and $\boldsymbol{P}_{\boldsymbol{U} \boldsymbol{A} \boldsymbol{V}}^{\boldsymbol{S}_{B H}}$, between two successive iterations, becomes negligible.

Having found the final value for the power $\boldsymbol{P}_{\boldsymbol{U} \boldsymbol{A} \boldsymbol{V}}^{\boldsymbol{S}_{B H}}$ for $S_{B H}$ subbands, in order to test the smaller value $\left(S_{B H}-1\right)$, the subband $s_{h}$ requiring the highest access power is removed from the backhaul subband pool before proceeding with the same described method.

These steps are summarized in Algorithm 1. The value of $S_{B H}$ requiring the minimum access power, $S_{B H}^{f}$, is retained. In case the total access power is smaller than $P_{U A V}^{\max }$, to maximize the intersection region, problem (17) is solved for the retained value, $S_{B H}^{f}$. Algorithm 1 also yields the backhaul subband assignment $\boldsymbol{b}$ and the intersection region $X_{S_{B H}^{f}}$. In the opposite case, i.e., when the total needed access power is larger than $P_{U A V}^{\max }$, the intersection region is chosen as the coverage disk of the MBS to guarantee the backhaul constraint. The complexity of Algorithm 1 stems from solving the convex problems (17), (20) and (24). While a closed form solution is found for (20) with a complexity in the order of $\mathcal{O}(S)$, (17) and (24) are solved using the interior point method, having $\mathcal{O}\left(S^{2.5}\right)$ as complexity [30]. Moreover, problem (17) is solved for the retained value of $S_{B H}$ requiring the minimum needed power, $S_{B H}^{f}$, while (20) and (24) are solved for all values of $S_{B H} \in \mathcal{S}_{B H}^{\text {poss }}$. Since $\left|\mathcal{S}_{B H}^{\text {poss }}\right| \leq S$, the complexity of Algorithm 1 is hence upper bounded by $\mathcal{O}\left(S^{3.5}\right)$.

\section{Finding the optimal UAV position and the final power levels}

To minimize the UAV transmit power, problem (5) is reformulated as follows:

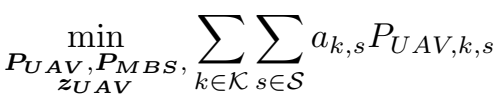




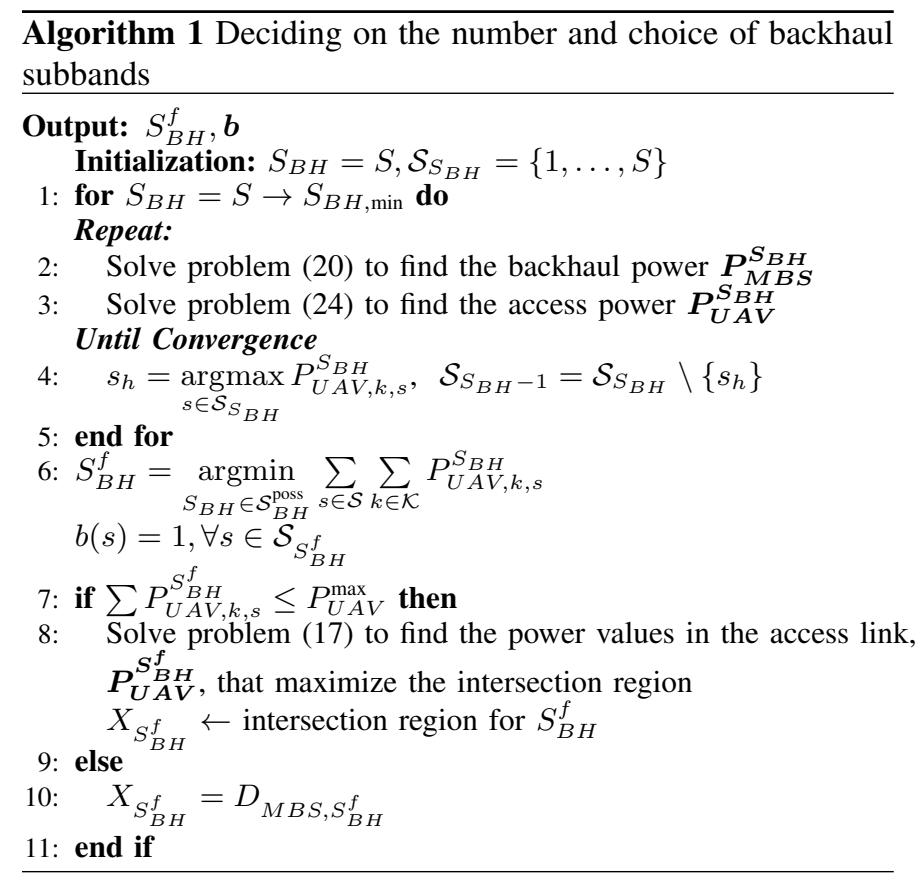

$$
\begin{aligned}
\text { such that } & \sum_{s \in \mathcal{S}} a_{k, s} R_{k, s}=R_{k}^{\mathrm{req}}, \forall k \in \mathcal{K}, \\
& \sum_{k \in \mathcal{K}} \sum_{s \in \mathcal{S}} a_{k, s} R_{k, s} \leq \sum_{s \in \mathcal{S}} b_{s} R_{U A V, s}, \\
& \sum_{s \in \mathcal{S}} b_{s} P_{M B S, s} \leq P_{M B S}^{\max }, \\
& H_{\min } \leq H \leq H_{\max }, \\
& \left(x_{U A V}, y_{U A V}\right) \in X_{S_{B H}^{f}} .
\end{aligned}
$$

Constraint (25e) states that the 2D position of the UAV should be in the simultaneous coverage region of the users and the MBS.

To minimize the needed access power, the BI should be minimized while meeting the backhaul rate requirement. To this end, the constraint (25b) should be met with equality.

From (3), $P_{U A V, k, s_{k}}$ satisfying (25a) for each user $k$ is:

$$
P_{U A V, k, s_{k}}=A_{1}(k)\left(N_{0} B_{c}+a_{s_{k}} P_{M B S, s_{k}} G_{k, s_{k}}^{2}\right) / G_{U A V, k}^{2},
$$

where $A_{1}(k)=\left(2^{R_{k}^{\text {req }} / B_{c}}-1\right)$ and $P_{M B S, s_{k}}$ is the backhaul power used by the MBS to meet $R_{U A V, s_{k}}$ given by:

$$
P_{M B S, s_{k}}=\left(2^{\frac{R_{U A V, s_{k}}}{B_{c}}}-1\right) \frac{N_{0} B_{c}+C_{S I} P_{U A V, k, s_{k}}}{G_{U A V, M B S}^{2}} .
$$

Note that in (26) and (27), the actual value of the elevation angle is used for the calculation of the channel gains $G$, i.e., the elevation angle of user $k$ and that of the MBS are given by: $\theta_{k}=\arctan \left(H / r_{k}\right)$ and $\theta_{M B S}=\arctan \left(H / r_{M B S}\right)$, respectively.

After replacing (27) in (26), $P_{U A V, k, s_{k}}$ can be expressed as:

$$
P_{U A V, k, s_{k}}=\frac{A_{1}(k) N_{0} B_{c}\left\{G_{U A V, M B S}^{2}+a_{s_{k}} A_{2}\left(s_{k}\right) G_{k, s_{k}}^{2}\right\}}{G_{U A V, M B S}^{2} G_{U A V, k}^{2}-a_{s_{k}} G_{k, s_{k}}^{2} A_{1}(k) A_{2}\left(s_{k}\right) C_{S I}},
$$

where $A_{2}\left(s_{k}\right)=\left(2^{R_{U A V, s_{k}} / B_{c}}-1\right)$.

Although $\boldsymbol{A}_{1}$ is known for all users, $\boldsymbol{A}_{\mathbf{2}}$ is not since it depends on the backhaul rate per subband which, in its turn, depends on the power in the backhaul and access links. Therefore, solving (25) consists of solving the following equivalent problem:

$$
\begin{aligned}
& \min _{\boldsymbol{z}_{U \boldsymbol{A} \boldsymbol{V}}, \boldsymbol{A}_{\mathbf{2}}} \sum_{k \in \mathcal{K}} P_{U A V, k, s_{k}} \\
& \text { such that } \sum_{k \in \mathcal{K}} \sum_{s \in \mathcal{S}} a_{k, s} R_{k, s}=\sum_{s \in \mathcal{S}} b_{s} R_{U A V, s}, \\
& \sum_{s \in \mathcal{S}} b_{s} P_{M B S, s} \leq P_{M B S}^{\max }, \\
& H_{\min } \leq H \leq H_{\max }, \\
&\left(x_{U A V}, y_{U A V}\right) \in X_{S_{B H}^{f}},
\end{aligned}
$$

where $P_{U A V, k, s_{k}}$ is given by (28).

The solution of (29) is obtained iteratively. At the first iteration, $\boldsymbol{A}_{\mathbf{2}}$ is initialized with an equal rate repartition in the backhaul link, leading to $A_{2}\left(s_{k}\right)=\left(2 \frac{R^{\text {req }}}{S_{B H^{f}}^{B_{c}}}-1\right), \forall s_{k} \in$ $\mathcal{S}$ such that $b\left(s_{k}\right)=1$. With $\boldsymbol{A}_{2}$ known, (29) is solved numerically for the UAV position. Then, with the computed position, the values of $\boldsymbol{A}_{2}$ minimizing the UAV transmit power are found. The two steps are iterated until convergence, reached when the change in the values of $z_{U A V}$ and $A_{2}$, between two consecutive iterations, becomes negligible. Upon convergence, the power values in the backhaul and access links are computed according to (27) and (28), respectively. The complexity of solving (29) is upper bounded by $\mathcal{O}\left(S^{2.5}\right)$ [30].

It should be noted that, in practice, once the UAV has been positioned, it can exchange the channel state information with its users and with the MBS. Hence, in reality, the final power values can be calculated with the actual channel state values instead of the mean channel state obtained by the probabilistic path loss model. It should also be noted that finding the UAV position by solving problem (29) and enforcing the horizontal position of the UAV to be in the intersection region of the coverage disks of all users as in constraint (29d) does not guarantee that the power needed by the UAV is lower than its power budget with probability one. In fact, it may not be possible to find a value of the UAV altitude $H$ simultaneously guaranteeing the rate requirements of all users. In that case, the actual achieved elevation angles differ significantly from the optimal value $\theta_{\text {opt }}$, leading to a higher needed power. If that should happen, the technique introduced next, in Section VI, is employed to maximize the achieved rates while respecting the UAV power budget.

\section{Case Where the Rate Requirements Cannot Be Met With $P_{U A V}^{\mathrm{MAX}}$}

If the user rate requirements are very high, the UAV power budget might not be enough to ensure them. In this case, either the feasibility test in (13) fails to find power values respecting the UAV budget, or Algorithm 1 fails to find a value of $S_{B H}$ respecting this budget. Moreover, in a small percentage of the cases, a value of the UAV altitude simultaneously guaranteeing the user rate requirements might not be achievable. If that should happen, the UAV position and the power values are still found according to the analysis of Section V-C. However, 
the solution of (29) violates constraint $(5 \mathrm{c})$. Therefore, additional steps, described hereinafter, are performed to enhance user satisfaction: After finding the maximum achievable user rates with the UAV power budget, a NOMA pairing step is conducted to increase, to the extent of the possible, the rates of users not yet reaching their target value.

\section{A. Finding the Maximum Achievable Rates with $P_{U A V}^{\max }$}

To find the maximum achievable rates respecting the UAV power budget, problem (30) is formulated:

$$
\begin{gathered}
\max _{\boldsymbol{P}_{\boldsymbol{U} A V}} \sum_{k \in \mathcal{K}} R_{k, s_{k}} \\
\text { such that } \sum_{k \in \mathcal{K}} P_{U A V, k, s_{k}} \leq P_{U A V}^{\max }, \\
\sum_{k \in \mathcal{K}} P_{M B S, s_{k}} \leq P_{M B S}^{\max }, \\
R_{k, s_{k}} \leq R_{k}^{\mathrm{req}}, \forall k \in \mathcal{K},
\end{gathered}
$$

where $R_{k, s_{k}}$ is the rate of user $k$ over its assigned subband $s_{k}$, given by Eq. (3). Constraint (30c) ensures that none of the users exceed their rate requirement. The power value $P_{M B S, s_{k}}$ is expressed using (27), with the value of $R_{U A V, s_{k}}$ found by the solution of problem (29). Problem (30) is convex, hence can be solved efficiently by the interior point method.

\section{B. Finding the Candidate Subbands for NOMA Pairing}

The solution of (30) yields the data rates that users can obtain from orthogonal multiple access (OMA) scheduling. To bring users closer to their requested rates, a NOMA pairing step is conducted to use the allocated power of unsatisfied users more efficiently.

1) NOMA Basics: With NOMA, the signals of up to $N_{n}$ users are superposed and transmitted over subband $n$. This multiplexing results in co-channel interference between the collocated users. Hence, user $j$, scheduled over $n$, performs SIC to remove the interference of some of the other collocated users before demodulating its own signal. The achievable rate in this case can be written as:

$$
R_{j, n}=B_{c} \log _{2}\left(1+\frac{P_{U A V, j, n} G_{U A V, j}^{2}}{I_{j, n}+b_{n} P_{M B S, n} G_{j, n}^{2}+N_{0} B_{c}}\right)
$$

where $I_{j, n}=\sum_{k \in \mathcal{I}_{j, n}} P_{U A V, k, n} G_{U A V, j}^{2}$ represents the cochannel interference experienced by user $j$ from users in the set $\mathcal{I}_{j, n}$, i.e., from users whose interference could not be removed. Because of the complexity resulting from SIC decoding, the maximum value of $N_{n}$ is restricted to $2, \forall n \in \mathcal{S}$. When $b_{n}=1$, due to the presence of the BI (second term in the denominator of (31)), user $j$, called first user, can remove the interference of the other user $k$ collocated on $n$, if their channel gains verify the following condition, proved in [31]:

$$
G_{U A V, k} G_{j, n}<G_{U A V, j} G_{k, n} .
$$

User $k$, called second user, decodes its signal directly while considering the signal of the first user $j$ as noise.

To guarantee SIC stability [19], [32], i.e., successful decoding at the level of user $j$, the signal of user $k$ must be received at user $j$ with an amount of power superior to that of $j$, added to the BI power at $j$. Indeed, as shown in [33], in case this power condition is not satisfied, the users outage probabilities will always be one. This condition is written as [31]:

$$
P_{U A V, k, s_{k}}>P_{U A V, j, n}+P_{M B S, n} G_{j, n}^{2} / G_{U A V, j}^{2} .
$$

When $b_{n}=0$, conditions (32) and (33) become respectively (34) and (35) as in the classical NOMA case [19], [32]:

$$
\begin{gathered}
G_{U A V, k}<G_{U A V, j}, \\
P_{U A V, k, s_{k}}>P_{U A V, j, n} .
\end{gathered}
$$

2) Determining the eligible subbands for each user: The set of users being considered for NOMA pairing, $\mathcal{K}_{N O M A}$, consists of users that have not achieved their requested data rates, i.e., $\mathcal{K}_{\text {NOMA }}=\left\{k \in \mathcal{K}, \mid R_{k}<R_{k}^{\text {req }}\right\}$. The set of subbands considered for NOMA pairing, $\mathcal{S}_{\text {NOMA }}$, consists of subbands belonging to users having achieved their required data rates, i.e., $\mathcal{S}_{\text {NOMA }}=\left\{s \in \mathcal{S} \mid a_{k, s}=1, R_{k}=R_{k}^{\text {req }}\right\}$. To bring users closer to their requested data rates, the power allocated to user $k \in \mathcal{K}_{N O M A}$ is divided between its originally allocated subband $s_{k}$ and a subband $n$ on which $k$ can be NOMA paired in a way to increase its throughput. To avoid penalizing users having achieved their rate requirements and to avoid having a chain of power modifications on each subband $n$ considered for NOMA pairing, we make the following assumptions:

1) unsatisfied users are paired as second users via NOMA,

2) the access power of the user already scheduled on $n$ (i.e., first user) is kept constant after pairing,

3) the backhaul power on subband $n$ is kept constant.

Assumption 1 ensures that the satisfied first user, initially scheduled on $n$, performs SIC in order to decode its message without the interference from the newly paired NOMA user. Together with assumptions 2 and 3, assumption 1 guarantees that the rate of the initially scheduled user is not penalized.

Let $k$ be an unsatisfied user initially scheduled as OMA user on subband $s_{k}$. Among $\mathcal{S}_{N O M A}, k$ can be scheduled via NOMA on a subset of subbands, denoted by $\mathcal{S}_{N O M A}^{k}$. Let $n \in \mathcal{S}_{N O M A}^{k}$ and let $j$ be the user already scheduled on $n$. Subband $n$ should satisfy the following conditions:

1) (34) when $b_{n}=0$ or (32) when $b_{n}=1$,

2) (35) when $b_{n}=0$, or (33) when $b_{n}=1$,

3) scheduling user $k$ on $n \in \mathcal{S}_{\text {NOMA }}^{k}$ can increase its achieved data rate,

4) scheduling user $k$ on $n \in \mathcal{S}_{N O M A}^{k}$ does not penalize the backhaul rate already achieved on subbands $n$ and $s_{k}$.

While finding subbands ensuring conditions 1 and 2 is straightforward, to guarantee the conditions 3 and 4, the following problem is solved:

$$
\min _{R_{k, s_{k}}^{\prime}, R_{k, n}^{\prime}} P_{U A V, k, s_{k}}^{\prime}+P_{U A V, k, n}^{\prime}
$$


such that $R_{k, s_{k}}^{\prime}+R_{k, n}^{\prime}=R_{k, s_{k}}$,

$$
\begin{aligned}
& P_{U A V, k, n}^{\prime}>P_{U A V, j, n}^{\prime} \text { if } b_{n}=0 \text { or } \\
& P_{U A V, k, n}^{\prime}>P_{U A V, j, n}+P_{M B S, n} G_{j, n}^{2} / G_{U A V, j}^{2} \\
& \text { if } b_{n}=1, \\
& P_{M B S, s_{k}}^{\prime} \leq P_{M B S, s_{k}}, \\
& R_{U A V, s_{k}}^{\prime}+R_{U A V, n}^{\prime}=R_{U A V, s_{k}}+R_{U A V, n}
\end{aligned}
$$

In (36), $P_{U A V, k, s_{k}}^{\prime}$ and $P_{U A V, k, n}^{\prime}$ are the power values relative to the signal of user $k$ on subbands $s_{k}$ and $n$ respectively whereas $R_{k, s_{k}}^{\prime}\left(R_{k, n}^{\prime}\right.$ resp.) is the updated rate of user $k$ on $s_{k}$ ( $n$ resp.). Constraint (36c) guarantees that $P_{M B S, s_{k}}^{\prime}$, the updated backhaul power on $s_{k}$, does not exceed its initial value $P_{M B S, s_{k}}$. Constraint (36d) guarantees that the sum backhaul rate on $s_{k}$ and $n$ remains constant, $R_{U A V, s_{k}}^{\prime}$ and $R_{U A V, n}^{\prime}$ being the updated backhaul rates on $s_{k}$ and $n$, respectively.

Solving (36) yields the minimum power values necessary to ensure the achievable rate of user $k$ (found by (30)), when paired over subband $n$ as second user. If this solution satisfies $\Delta P=P_{U A V, k, s_{k}}-\left(P_{U A V, k, s_{k}}^{\prime}+P_{U A V, k, n}^{\prime}\right) \geq 0$, user $k$ can use the excess power $\Delta P$ to increase its achieved rate. To solve (36), the cases where subband $n$ is used in the backhaul link or not, are discussed separately in Appendix B.

\section{NOMA Pairing and Power Optimization}

In this section, the assignment of unsatisfied users to the candidate subbands, as well as the power optimization subsequent to pairing are discussed.

To conduct the NOMA pairing step, a metric $M(k, n)$ is associated with each pair $\left(k \in \mathcal{K}_{N O M A}, n \in \mathcal{S}_{\text {NOMA }}^{k}\right)$, depending on whether $n$ is used in the backhaul link or not.

$$
M(k, n)= \begin{cases}\frac{P_{U A V, j, n} G_{U A V, k}^{2}+P_{M B S, n} G_{k, n}^{2}}{G_{U A V, k}^{2}}, & \text { if } b_{n}=1, \\ -G_{U A V, k}^{2}, & \text { otherwise. }\end{cases}
$$

In the case where $b_{n}=1, M(k, n)$ reflects the $\mathrm{BI}$ as well as the NOMA interference suffered by user $k$ (Cf. Eq. (31)), if paired on subband $n$. If $b_{n}=0$, the metric takes into account only the channel gain of user $k$. By minimizing $M(k, n)$, the achieved rate is maximized by minimizing the interference when $b_{n}=1$ and by maximizing the channel gain of the paired user when $b_{n}=0$.

Once this metric is calculated, the Hungarian algorithm is used to find the NOMA assignment of unsatisfied users to candidate subbands. Then, the following optimization problem is solved for each paired user separately, to find the new power values and deduce the achieved user rate:

$$
\begin{gathered}
\max _{\substack{P_{U A V, k, s_{k}}^{\prime} \\
P_{U A V, k, n}^{\prime}}} R_{k, s_{k}}^{\prime}+R_{k, n}^{\prime} \\
\text { such that } P_{U A V, k, s_{k}}^{\prime}+P_{U A V, k, n}^{\prime} \leq P_{U A V, k, s_{k}} \\
P_{U A V, k, n}^{\prime}>P_{U A V, j, n}^{\prime} \text { if } b_{n}=0 \text { or } \\
P_{U A V, k, n}^{\prime}>P_{U A V, j, n}+P_{M B S, n} G_{j, n}^{2} / G_{U A V, j}^{2} \\
\text { if } b_{n}=1, \\
P_{M B S, s_{k}}^{\prime} \leq P_{M B S, s_{k}}
\end{gathered}
$$

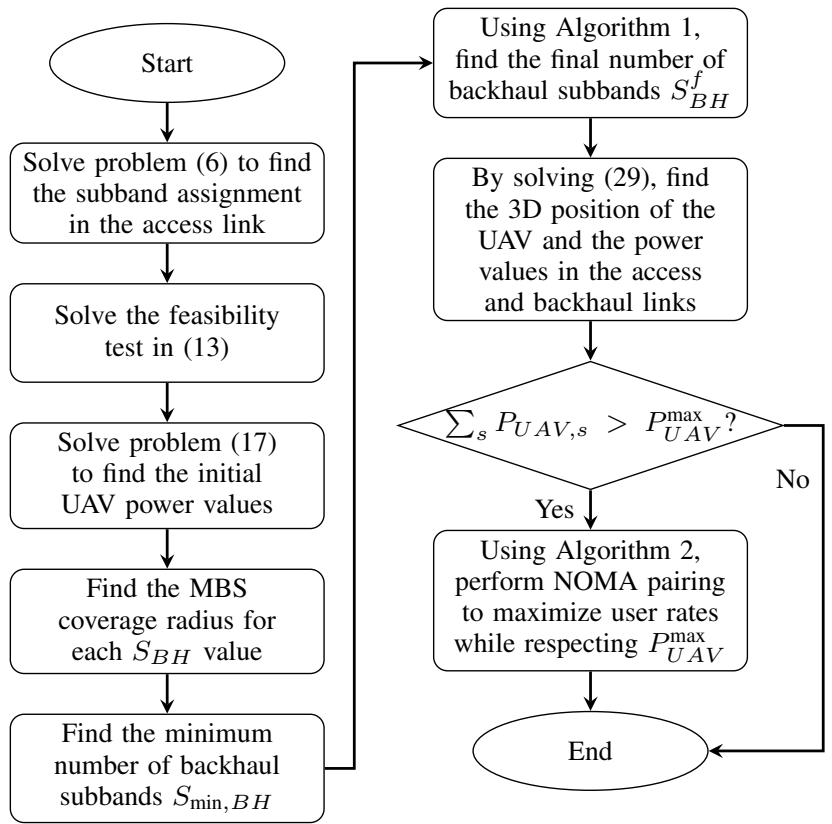

Fig. 2: Flow chart of the proposed method.

$$
\begin{aligned}
& R_{U A V, s_{k}}^{\prime}+R_{U A V, n}^{\prime}=R_{U A V, s_{k}}+R_{U A V, n} \\
& R_{k, s_{k}}^{\prime}+R_{k, n}^{\prime} \leq R_{k}^{\text {req }} .
\end{aligned}
$$

Problem (38) divides the total power allocated to user $k$, $P_{U A V, k, s_{k}}$, between subbands $s_{k}$ and $n$, to maximize its achieved rate.

The steps described in section VI-C are repeated until either all users are satisfied, or no more NOMA pairings can take place. The following algorithm summarizes the additional steps described in section VI:

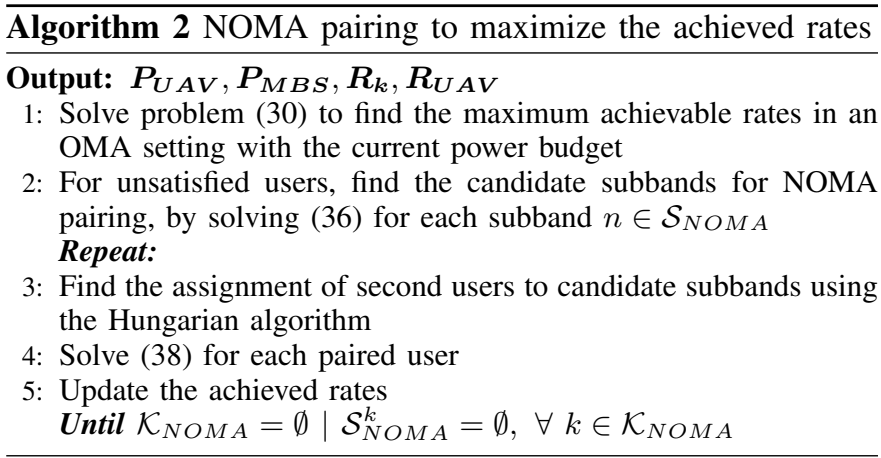

The flowchart in Fig. 2 summarizes the overall proposed method.

\section{Simulation Results}

Extensive simulations of the proposed framework were conducted, where users are randomly located within a squared urban area of size $1 \mathrm{Km} \times 1 \mathrm{Km}$. The MBS is located at the bottom left corner, as shown in Fig. 1. The simulation parameters are listed in Table I, with the urban environment constants set as in [27]. Users are divided into two different QoS classes. For $K=32$, the first class corresponds to rate requirements from 4 to $4.8 \mathrm{Mbps}$ and the second ranging from 9 to 9.8 Mbps. 
TABLE I: Simulation Parameters

\begin{tabular}{|c|c|c|c|}
\hline Parameter & Value Range & Parameter & Value Range \\
\hline$(\alpha, \beta)$ & $(9.61,0.16)$ & $\left(\eta_{L}, \eta_{N L}\right)$ & $(1,20) \mathrm{dB}$ \\
\hline$f_{c}$ & $2 \mathrm{GHz}$ & $B W$ & $20 \mathrm{MHz}$ \\
\hline$P_{M B S}^{\max }$ & 2 to $8 \mathrm{~W}$ & $H_{\min }$ & $100 \mathrm{~m}$ \\
\hline$P_{U A V}^{\max }$ & 0.5 to $3 \mathrm{~W}$ & $H_{\max }$ & $800 \mathrm{~m}$ \\
\hline$K$ & $8,16,32,64$ & $R^{\text {req }}=\sum_{k} R_{k}^{\text {req }}$ & $\begin{array}{c}208 \text { to } \\
233.6 \mathrm{Mbps}\end{array}$ \\
\hline $\begin{array}{c}\text { User-MBS } \\
\text { Path Loss }\end{array}$ & $\begin{array}{c}128.1+37.6 \times \\
\log _{10}(d[K m])\end{array}$ & $\begin{array}{c}\text { RMS delay } \\
\text { spread }\end{array}$ & $500 \mathrm{~ns}$ \\
\hline $1 / C_{S I}$ & $130 \mathrm{~dB}$ & $N_{0}$ & $-174 \mathrm{dBm} / \mathrm{Hz}$ \\
\hline
\end{tabular}

The method proposed in this study is denoted by OptPInit\&MinP-NOMA. The performance of a variant of this method that does not perform the NOMA pairing step detailed in Algorithm 2 is also shown, and is denoted by OptPInit\&MinP-OMA. For comparison, an algorithm based on the study in [11], denoted by OBA-PSO, is also evaluated. In OBA-PSO, the available frequency band is divided orthogonally between the access and backhaul links and the 3D placement of the UAV is conducted using the particle swarm optimization (PSO) algorithm. In more detail, the amount of spectrum needed to achieve the backhaul rate requirement is first determined. Then, the remaining amount of bandwidth is divided into $K$ subbands, before assigning each subband to one user. The performance of the technique formerly proposed in [26], denoted by EqPInit\&MaxIntSubAss, is also shown for comparison. EqPInit\&MaxIntSubAss also considers an IBFD wireless backhaul system, but assumes an equal initial power repartition on the access and backhaul subbands. Also, the number of backhaul subbands is chosen to maximize the simultaneous coverage region. Moreover, when the method in [26] fails to find a solution that respects the UAV power budget, the rate requirement of all users is gradually reduced by the amount $\delta R=0.25 \mathrm{Mbps}$, until a solution is found.

\section{A. Convergence of the Proposed Technique}

First, the convergence of both the iterative method of Algorithm 1 and problem (29) is analyzed. Fig. 3 shows the number of needed iterations before convergence is reached in both Algorithm 1 and problem (29).

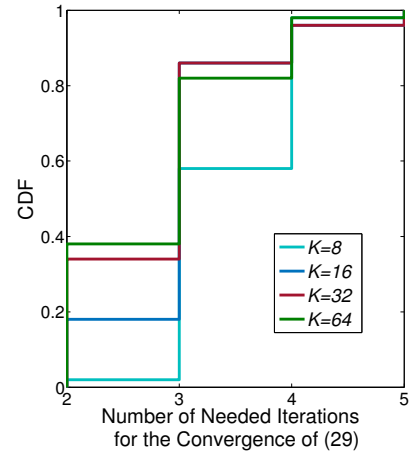

(a)

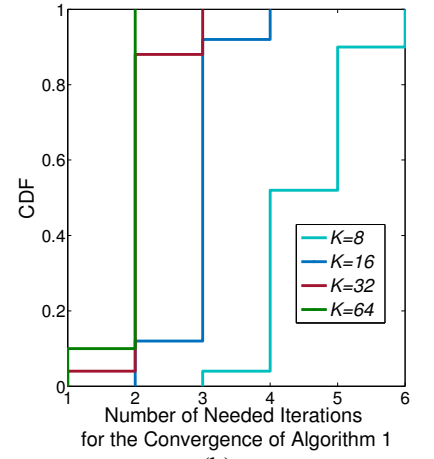

(b)
Fig. 3: Number of needed iterations for the convergence of (a) problem (29), (b) Algorithm 1.

In Fig. 3a, the cumulative distribution function (CDF) of the number of needed iterations for the convergence of problem (29) is plotted for various values of $K$. Fig. 3a shows that problem (29) converges within a very small number of iterations (less than 5) for $K=8,16,32$ and 64 users, hence, fairly quickly. Fig. 3b plots the CDF of the number of needed iterations before Algorithm 1 converges for various values of $K$. Fig. 3b also shows that the iterative approach of Algorithm 1 converges within a very small number of iterations (less than 6) for all $K$ values.

\section{B. Performance of the Proposed Technique}

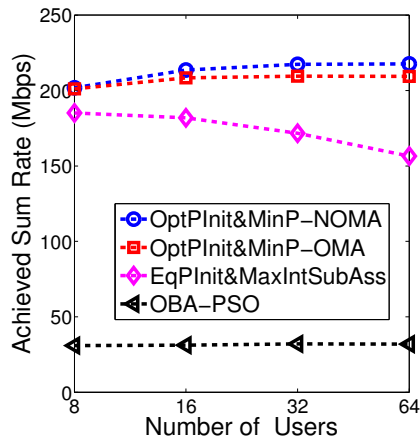

(a)

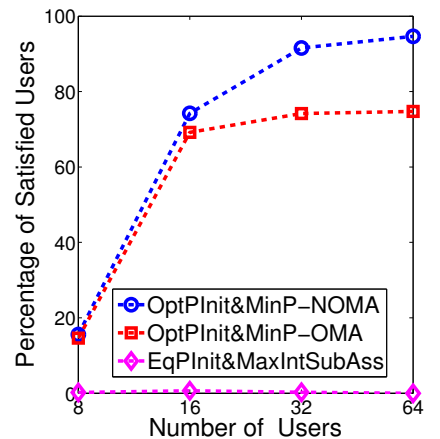

(b)
Fig. 4: Achieved sum rate (a), and percentage of satisfied users (b), in terms of $K$ for $R^{\text {req }}=220 \mathrm{Mbps}, P_{U A V}^{\max }=1 \mathrm{~W}$ and $P_{M B S}^{\max }=4 W$.

Fig. 4 compares the performance of the different methods for $K$ ranging between 8 and 64 . It was assumed hat $75 \%$ of the users have a sum rate requirement of $132 \mathrm{Mbps}$ while the remaining $25 \%$ have a sum rate requirement of $88 \mathrm{Mbps}$, resulting in $R^{\text {req }}=220 \mathrm{Mbps}, \forall K$. Fig. 4 a shows that OBAPSO results in the lowest achieved sum rate. In fact, because of the spectrum division between the access and the backhaul links, the amount of bandwidth assigned to each user is inherently smaller than in our proposed method. Constrained by the UAV power budget, users cannot be served with a sufficient rate. In contrast, our proposed methods based on IBFD wireless backhauling achieve much higher data rates. However, EqPInit\&MaxIntSubAss achieves the lowest data rate among these methods because of the initial equal power repartition and the reduction of data rates when $P_{U A V}^{\max }$ is not sufficient to satisfy user requirements. As $K$ increases, the achieved throughput of EqPInit\&MaxIntSubAss deteriorates because the data rate of each user is reduced by a constant amount of $\delta R$. On the other hand, the achieved throughput of both OptPInit\&MinP-OMA and OptPInit\&MinP-NOMA increases with $K$ because they can better exploit multiuser diversity, with OptPInit\&MinP-NOMA outperforming its OMA counterpart. At $K=64$, OptPInit\&MinP-NOMA outperforms OptPInit\&MinP-OMA, EqPInit\&MaxIntSubAss and OBA-PSO by 8, 61 and $186 \mathrm{Mbps}$, respectively. When it comes to the percentage of users having received their data rate, Fig. 4b shows that EqPInit\&MaxIntSubAss can hardly satisfy any user. The performance of OBA-PSO is not shown since the satisfaction percentage is $0 \%$ for all values of $K$. OptPInit\&MinP-NOMA achieves an average satisfaction percentage of $94 \%$ for $K=64$, outperforming OptPInit\&MinPOMA by almost $20 \%$, which shows the benefit of the proposed NOMA pairing. Note that the low value of the satisfaction 
percentage at $K=8$ is due to the fact that since the number of subbands $S$ is equal to $K$, when $S$ is too low, the amount of BI present at the quasi-totality of subbands prevents most users from achieving their target. Moreover, since $R^{\text {req }}=220$ Mbps $\forall K$, the rate requirement per user is higher for smaller values of $K$.

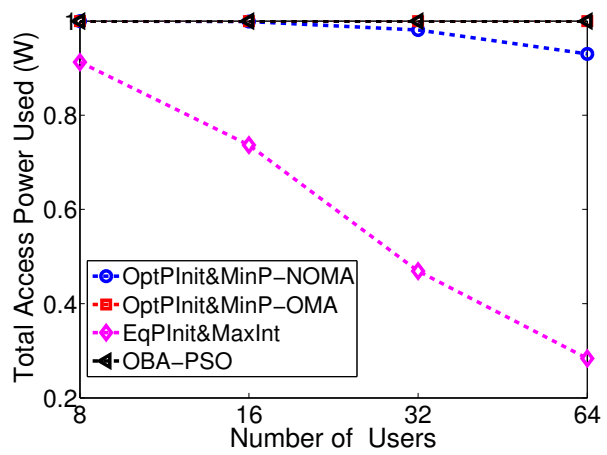

Fig. 5: Used access power in terms of $K$ for $R^{\text {req }}=220 \mathrm{Mbps}$, $P_{U A V}^{\max }=1 \mathrm{~W}$ and $P_{M B S}^{\max }=4 \mathrm{~W}$.

In Fig. 5, the needed UAV transmit power is shown in terms of $K$. The power for EqPInit\&MaxIntSubAss is a decreasing function of $K$ because the achieved rate also decreases with $K$ for this method. Both OptPInit\&MinP-OMA and OBA-PSO consume the total budget to maximize the achieved rate. On the other hand, the needed power for OptPInit\&MinP-NOMA is lower than that of its OMA counterpart when $K=32$ or 64 users. Hence, the NOMA pairing step is not only able to increase the achieved data rate, but can do so while consuming less transmit power.

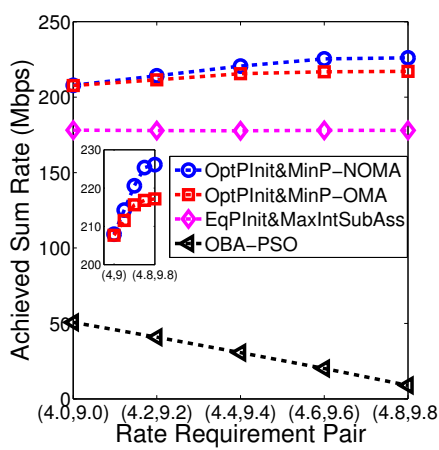

(a)

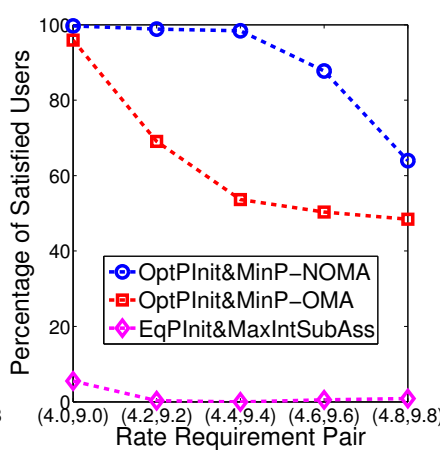

(b)
Fig. 6: Achieved sum rate (a), and percentage of satisfied users (b), in terms of $R_{k}^{\mathrm{req}}$ for $K=32, P_{U A V}^{\max }=1 \mathrm{~W}$ and $P_{M B S}^{\max }=4 \mathrm{~W}$.

Fig. 6 compares the performance of the different methods for $K=32$ users with a varying data rate requirement. The $K$ users are equally partitioned into 2 classes based on their data rate requirements, shown on the x-axis of Fig. $6 \mathrm{a}$ and $6 \mathrm{~b}$. As the target rate increases, the achieved rate of OBA-PSO decreases as more bandwidth is needed to meet the backhaul rate requirement. OBA-PSO is also unable to satisfy any user. OptPInit\&MinP-OMA and OptPInit\&MinP-NOMA achieve the best performance, with OptPInit\&MinP-NOMA outperforming OptPInit\&MinP-OMA and EqPInit\&MaxIntSubAss by up to 9 and $48 \mathrm{Mbps}$, respectively in terms of data rate, and up to $45 \%$ and $98 \%$, respectively in terms of percentage of satisfaction.

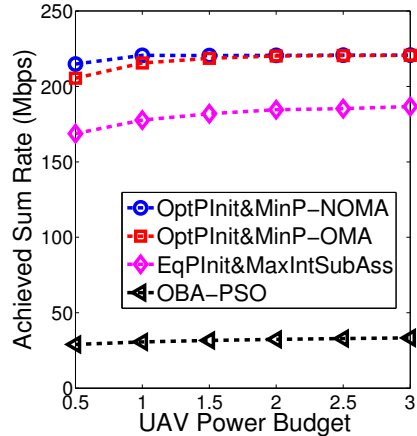

(a) (b)

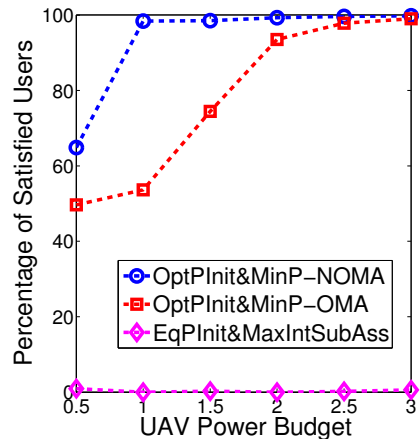

Fig. 7: Achieved sum rate (a), and percentage of satisfied users (b), in terms of $P_{U A V}^{\max }$ for $K=32$ and $P_{M B S}^{\max }=4 W$.

Fig. 7 compares the performance of the different methods for a varying $P_{U A V}^{\max }$. The number of users is $K=32$, half of which have a rate requirement of $4.4 \mathrm{Mbps}$, while the other half requires $9.4 \mathrm{Mbps}$. Although the rate achieved by EqPInit\&MaxIntSubAss increases with $P_{U A V}^{\max }$, its percentage of satisfaction remains very low. OptPInit\&MinP-NOMA outperforms the OMA version when $P_{U A V}^{\max }$ is low, however the gap diminishes when the latter increases as OMA scheduling has a higher chance of satisfying all users.

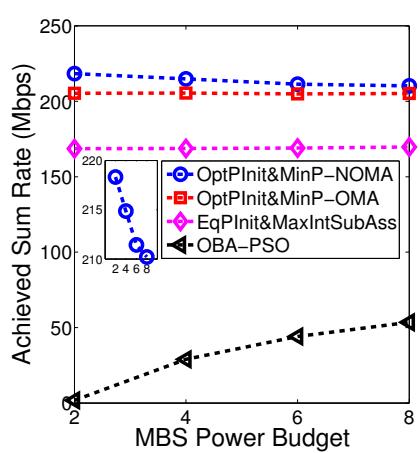

(a)

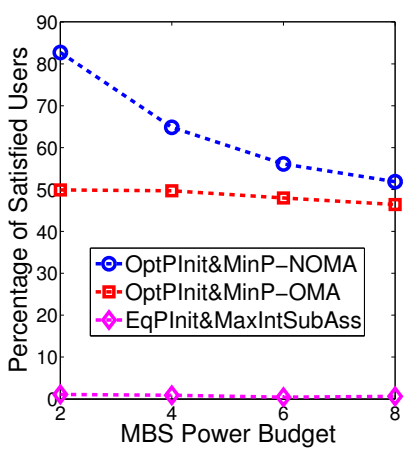

(b)
Fig. 8: Achieved sum rate (a), and percentage of satisfied users (b), in terms of $P_{M B S}^{\max }$ for $K=32$ and $P_{U A V}^{\max }=0.5 \mathrm{~W}$.

Finally, Fig. 8 compares the performance of the different methods in terms of $P_{M B S}^{\max }$, for $K=32$ users with the same rate requirements as in Fig. 7. The rate achieved by OBAPSO increases with $P_{M B S}^{\max }$ as the amount of needed spectrum for the backhaul link decreases, allowing users a larger bandwidth. However, OBA-PSO still cannot satisfy any user. On the other hand, the achieved rate of OptPInit\&MinP-NOMA decreases with $P_{M B S}^{\max }$. This is due to NOMA being less beneficial when a higher $\mathrm{BI}$, resulting from a higher $P_{M B S}^{\max }$, is experienced. Nonetheless, OptPInit\&MinP-NOMA still outperforms OptPInit\&MinP-OMA, EqPInit\&MaxIntSubAss and OBA-PSO by up to 13,50 , and 217 Mbps respectively, in terms of throughput, and by up to 33,81 , and $83 \%$ in terms of user satisfaction.

\section{CONCLUSION}

A new technique to optimize the resource allocation and 3D placement in UAV-enabled networks while considering the backhaul connectivity of the UAV-BS was introduced. 
Accounting for the backhaul capacity and the power budgets of the UAV and the MBS, the proposed technique aims at minimizing the UAV transmit power necessary to meet the user rate requirements. It benefits from both, an in-band full duplex wireless backhaul, and NOMA pairing to boost system performance. The results showed that the proposed approach significantly increases the achieved throughput and the percentage of satisfied users, compared to the case of a UAV-enabled network that uses separate frequency bands in the access and backhaul links, and also towards a previously proposed method. Moreover, the technique proposed in this work can be easily extended to study other network settings, such as the deployment of pico-cell BSs.

\section{APPENDIX A}

As seen from (9), the MBS coverage radius $C_{M B S, S_{B H}}$ increases when $L_{S_{B H}}^{\text {th }}$, given by (18), does. In addition, $L_{S_{B H}}^{\text {th }}$ increases when the term inside the brackets in (18) decreases. In other words, $L_{S_{B H}}^{\text {th }}$ increases when (39) decreases:

$$
F\left(S_{B H}\right)=\left(2^{\frac{R^{\mathrm{req}}}{S_{B H} \times B_{c}}}-1\right) \times S_{B H} / P_{M B S}^{\max } .
$$

The first derivative of $F\left(S_{B H}\right)$ with respect to $S_{B H}$ is:

$$
\frac{\partial F}{\partial S_{B H}}=\frac{1}{P_{M B S}^{\max }}\left(2^{\frac{R^{\mathrm{req}}}{S_{B H} \times B_{c}}}\left(1-\frac{R^{\mathrm{req}} \log (2)}{S_{B H} \times B_{c}}\right)-1\right) \text {. }
$$

Hence, $F$ decreases with $S_{B H}$ if:

$$
g\left(S_{B H}\right)=2^{\frac{R^{\text {req }}}{S_{B H} \times B_{C}}}\left(1-\frac{R^{\text {req }} \log (2)}{S_{B H} \times B_{C}}\right)<1 .
$$

The first derivative of $g\left(S_{B H}\right)$ with respect to $S_{B H}$ is:

$$
\frac{\partial g}{\partial S_{B H}}=\frac{\left(R^{\text {req }}\right)^{2} \log (2)^{2}}{\left(S_{B H}\right)^{3}\left(B_{c}\right)^{2}} \times 2^{\frac{R_{B H}^{\text {req }}}{S_{B B_{c}}}}>0,
$$

i.e., $g\left(S_{B H}\right)$ is strictly increasing. Moreover, $\lim _{S_{B H} \rightarrow \infty} g\left(S_{B H}\right)=1$. Hence, (41) is always guaranteed and $F$ is a decreasing function of $S_{B H}$. Thus, $C_{M B S, S_{B H}}$ increases with $S_{B H}$.

\section{APPENDIX B}

\section{A. Subband $n$ is not used in the backhaul link}

In this case, constraint (36d) comes down to ensuring $R_{U A V, s_{k}}^{\prime}=R_{U A V, s_{k}}$. If $b_{s_{k}}=0$, i.e., $s_{k}$ is not used in the backhaul link, (36d) is automatically satisfied. On the other hand, if $b_{s_{k}}=1$, with the decrease of $P_{U A V, k, s_{k}}^{\prime}$ resulting from the division of $P_{U A V, k, s_{k}}^{\prime}$ between subbands $s_{k}$ and $n$, the SI experienced by the UAV on $s_{k}$ decreases. To keep $R_{U A V, s_{k}}$ constant, hence ensuring (36d), $P_{M B S, s_{k}}^{\prime}$ should decrease as per Eq. (4). Therefore, constraint (36c) is always met when the total access power allocated to user $k$ decreases. According to (28), $P_{U A V, k, s_{k}}^{\prime}$ can be formulated as:

$$
P_{U A V, k, s_{k}}^{\prime}=\frac{\left(2^{R_{k, s_{k}}^{\prime} / B_{c}}-1\right) J_{1}}{J_{2}-J_{3}\left(2^{R_{k, s_{k}}^{\prime} / B_{c}}-1\right)} .
$$

where

$$
\left\{\begin{array}{l}
J_{1}=N_{0} B_{c}\left\{G_{U A V, M B S}^{2}+a_{k, s} A_{2}\left(s_{k}\right) G_{k, s_{k}}^{2}\right\}, \\
J_{2}=G_{U A V, M B S}^{2} G_{U A V, k}^{2}, \\
J_{3}=a_{k, s} G_{k, s_{k}}^{2} A_{2}\left(s_{k}\right) C_{S I} .
\end{array}\right.
$$

From (31), when $b_{n}=0$, the power needed on subband $n$ to achieve a rate of $R_{k, n}^{\prime}$ is found according to:

$$
P_{U A V, k, n}^{\prime}=\left(2^{R_{k, n}^{\prime} / B_{c}}-1\right) \times J_{4},
$$

where $J_{4}=N_{0} B_{c} / G_{U A V, k}^{2}+P_{U A V, j, n}$.

Let $\left(2^{R_{k, s_{k}}^{\prime} / B_{c}}-1\right)=x$. In order to ensure constraint (36a), $P_{U A V, k, n}^{\prime}$ should take the following value:

$$
P_{U A V, k, n}^{\prime}=\left(\frac{2^{R_{k, s_{k}} / B_{c}}}{x+1}-1\right) J_{4}
$$

Then, the total needed power to achieve $R_{k, s_{k}}$ is given by:

$$
\begin{aligned}
P_{k}^{\prime}(x) & =P_{U A V, k, s_{k}}^{\prime}(x)+P_{U A V, k, n}^{\prime}(x) \\
& =\frac{x J_{1}}{J_{2}-x J_{3}}+\left(\frac{2^{R_{k, s_{k}} / B_{c}}}{x+1}-1\right) J_{4} .
\end{aligned}
$$

$P_{k}^{\prime}$ is convex in $x$ as $\frac{\partial^{2} P_{k}^{\prime}}{\partial^{2} x}>0$. The value of $x$ that minimizes $P_{k}^{\prime}$ is found as:

$$
x^{*}=\frac{J_{2} \sqrt{J_{4} 2^{R_{k, s_{k}}}}-\sqrt{J_{1} J_{2}}}{J_{3} \sqrt{J_{4} 2^{R_{k, s_{k}}}}+\sqrt{J_{1} J_{2}}} .
$$

Having $x^{*}$, the minimum updated individual power values on $s_{k}$ and $n$ as well as the total user power are found using (43), (45) and (46), respectively. If these power values satisfy (36b), then $n$ is a valid candidate. In the opposite case, $x_{S I C}$, the value of $x$ that enforces (36b) at equality, is found, and $n$ is a valid candidate if $P_{k}^{\prime}\left(x_{S I C}\right)<P_{U A V, k, s_{k}}$. In fact, in the second case, we may have $x_{S I C}<x^{*}$ (in the decreasing part of $P_{k}^{\prime}(x)$ ) or $x_{S I C}>x^{*}$ (in the increasing part). But in both cases, $P_{k}^{\prime}\left(x_{S I C}\right)>P_{k}^{\prime}\left(x^{*}\right)$.

\section{B. Subband $n$ is used in the backhaul link}

The backhaul power on $n$ is kept constant, as per assumption 3. Therefore, when user $k$ is scheduled on subband $n$, the SI term in the denominator of (4) increases, leading to $R_{U A V, n}^{\prime}<R_{U A V, n}$. To ensure (36d), $R_{U A V, s_{k}}^{\prime}$ must be increased with respect to $R_{U A V, s_{k}}$ while respecting (36c). Hence, in this case, $R_{U A V, s_{k}}^{\prime}$ is not constant, and by extension neither is term $A_{2}\left(s_{k}\right)$ in (43). In fact, from (4), $R_{U A V, n}^{\prime}$ is a decreasing function of $P_{U A V, k, n}^{\prime}$ leading to $R_{U A V, s_{k}}^{\prime}$ and $A_{2}\left(s_{k}\right)$ being increasing functions of $P_{U A V, k, n}^{\prime}$. This leads to a set of non-linear expressions for $R_{U A V, s_{k}}^{\prime}$ and $R_{U A V, n}^{\prime}$. Hence, obtaining closed-form expressions of $R_{U A V, s_{k}}^{\prime}$ and $R_{U A V, n}^{\prime}$ that minimize the total needed power is cumbersome. Therefore, (36) is solved numerically to find whether pairing $k$ on $n$ can increase its total achieved rate.

It should be noted that due to the decrease of $R_{U A V, n}^{\prime}$, only subbands $s_{k}$, such that $b_{s_{k}}=1$ are considered when $b_{n}=1$.

\section{REFERENCES}

[1] Y. Zeng, R. Zhang, and T. J. Lim, "Wireless communications with unmanned aerial vehicles: opportunities and challenges," IEEE Commun. Mag., vol. 54, no. 5, pp. 36-42, May 2016. 
[2] E. Kalantari, M. Z. Shakir, H. Yanikomeroglu, and A. Yongacoglu, "Backhaul-aware robust 3D drone placement in 5G+ wireless networks," in Proc. Int. Conf. on Commun. (ICC) Workshops, Paris, France, May 2017, pp. 109-114.

[3] M. Mozaffari, W. Saad, M. Bennis, Y. Nam, and M. Debbah, "A tutorial on UAVs for wireless networks: Applications, challenges, and open problems," IEEE Commun. Surveys Tuts., pp. 1-28, Mar. 2019.

[4] L. Wang, B. Hu, and S. Chen, "Energy efficient placement of a drone base station for minimum required transmit power," IEEE Wireless Commun. Lett., Early Access, Feb. 2018.

[5] A. Al-Hourani, S. Kandeepan, and S. Lardner, "Optimal LAP altitude for maximum coverage," IEEE Wireless Commun. Lett., vol. 3, no. 6, pp. 569-572, Dec. 2014.

[6] M. Alzenad, A. El-Keyi, and H. Yanikomeroglu, "3-D placement of an unmanned aerial vehicle base station for maximum coverage of users with different QoS requirements," IEEE Wireless Commun. Lett., vol. 7, no. 1, pp. 38-41, Feb. 2018.

[7] M. Heino, D. Korpi, T. Huusari, E. Antonio-Rodriguez, S. Venkatasubramanian, T. Riihonen, L. Anttila, C. Icheln, K. Haneda, R. Wichman, and M. Valkama, "Recent advances in antenna design and interference cancellation algorithms for in-band full duplex relays," IEEE Commun. Mag., vol. 53, no. 5, pp. 91-101, May 2015.

[8] U. Siddique, H. Tabassum, E. Hossain, and D. I. Kim, "Wireless backhauling of 5G small cells: challenges and solution approaches," IEEE Wireless Commun., vol. 22, no. 5, pp. 22-31, Oct. 2015.

[9] Y. Choi and H. Shirani-Mehr, "Simultaneous transmission and reception: Algorithm, design and system level performance," IEEE Trans. Wireless Commun., vol. 12, no. 12, pp. 5992-6010, Dec. 2013.

[10] L. Zhang and N. Ansari, "On the number and 3-D placement of inband full-duplex enabled drone-mounted base-stations," IEEE Wireless Commun. Lett., vol. 8, no. 1, pp. 221-224, Feb. 2019.

[11] E. Kalantari, I. Bor-Yaliniz, A. Yongacoglu, and H. Yanikomeroglu, "User association and bandwidth allocation for terrestrial and aerial base stations with backhaul considerations," in Proc. IEEE Annual Int. Symp. on Personal, Indoor, and Mobile Radio Commun. (PIMRC), Montreal, QC, Canada, Oct. 2017, pp. 1-6.

[12] A. Benjebbour, A. Li, Y. Saito, Y. Kishiyama, A. Harada, and T. Nakamura, "System-level performance of downlink NOMA for future LTE enhancements," in Proc. IEEE Global Commun. Conf. (GLOBECOM) Workshops, Dec. 2013, pp. 66-70.

[13] M. J. Youssef, J. Farah, C. A. Nour, and C. Douillard, "Waterfillingbased resource allocation techniques in downlink non-orthogonal multiple access (NOMA) with single-user MIMO," in IEEE Symp. on Comput. and Commun. (ISCC), July 2017, pp. 499-506.

[14] K. Yang, N. Yang, N. Ye, M. Jia, Z. Gao, and R. Fan, "Non-orthogonal multiple access: Achieving sustainable future radio access," IEEE Commun. Mag., vol. 57, no. 2, pp. 116-121, Feb. 2019.

[15] Y. Sun, D. W. K. Ng, Z. Ding, and R. Schober, "Optimal joint power and subcarrier allocation for full-duplex multicarrier non-orthogonal multiple access systems," IEEE Trans. Commun., vol. 65, no. 3, pp. 1077-1091, Mar. 2017.

[16] S. Timotheou and I. Krikidis, "Fairness for non-orthogonal multiple access in 5G systems," IEEE Signal Process. Lett., vol. 22, no. 10, pp. $1647-1651$, Oct. 2015.

[17] L. Lei, D. Yuan, and P. Värbrand, "On power minimization for nonorthogonal multiple access (NOMA)," IEEE Commun. Lett., vol. 20, no. 12 , pp. $2458-2461$, Dec. 2016.

[18] J. Farah, E. Sfeir, C. A. Nour, and C. Douillard, "New resource allocation techniques for base station power reduction in orthogonal and nonorthogonal multiplexing systems," in Proc. Int. Conf. on Commun. (ICC) Workshops, May 2017, pp. 618-624.

[19] M. J. Youssef, J. Farah, C. A. Nour, and C. Douillard, "Resource allocation for mixed traffic types in distributed antenna systems using NOMA," Proc. IEEE Veh. Techn. Conf. Fall (VTC), Aug. 2018.

[20] — " "Resource Allocation in NOMA Systems for Centralized and Distributed Antennas with Mixed Traffic using Matching Theory," IEEE Trans. Commun., Early Access, 2019.

[21] J. Farah, A. Kilzi, C. A. Nour, and C. Douillard, "Power minimization in distributed antenna systems using non-orthogonal multiple access and mutual successive interference cancellation," IEEE Trans. Veh. Technol., vol. 67 , no. 12 , pp. $11873-11885$, Dec. 2018.

[22] T. M. Nguyen, W. Ajib, and C. Assi, "A novel cooperative NOMA for designing UAV-assisted wireless backhaul networks," IEEE J. Sel. Areas Commun., vol. 36, no. 11, pp. 2497-2507, Nov. 2018.

[23] Y. Liu, Z. Qin, Y. Cai, Y. Gao, G. Y. Li, and A. Nallanathan, "UAV communications based on non-orthogonal multiple access," IEEE Wireless Commun., vol. 26, no. 1, pp. 52-57, Feb. 2019.
[24] A. A. Nasir, H. D. Tuan, T. Q. Duong, and H. V. Poor, "UAV-Enabled Communication Using NOMA," IEEE Trans. Commun., vol. 67, no. 7, pp. 5126-5138, July 2019.

[25] N. Zhao, X. Pang, Z. Li, Y. Chen, F. Li, Z. Ding, and M. Alouini, "Joint trajectory and precoding optimization for UAV-Assisted NOMA Networks," IEEE Trans. on Commun., vol. 67, no. 5, pp. 3723-3735, May 2019.

[26] M. J. Youssef, C. A. Nour, J. Farah, and C. Douillard, "Backhaulconstrained resource allocation and 3D placement for UAV-enabled networks," Proc. IEEE Veh. Techn. Conf. Fall (VTC), Sept. 2019.

[27] A. Al-Hourani, S. Kandeepan, and A. Jamalipour, "Modeling air-toground path loss for low altitude platforms in urban environments," in Proc. IEEE Global Commun. Conf. (GLOBECOM), Austin, Tx, USA, Dec. 2014, pp. 2898-2904.

[28] J. Munkres, "Algorithms for the assignment and transportation problems," in Journal of the Society for Industrial and Applied Mathematics, vol. 5, 1957, pp. 32-38.

[29] J. Eckhoff, "Helly, Radon, and Carathéodory type theorems," in Handbook of Convex Geometry, North-Holland, 1993, pp. 389-448.

[30] P. M. Vaidya, "Speeding-up linear programming using fast matrix multiplication," in 30th Annual Symposium on Foundations of Computer Science, Oct 1989, pp. 332-337.

[31] J. Farah, J. Akiki, and E. P. Simon, "Energy-efficient techniques for combating the influence of reactive jamming using non-orthogonal multiple access and distributed antenna systems," Wireless Telecom. Symp. (WTS), Apr. 2019

[32] J. Zhu, J. Wang, Y. Huang, S. He, X. You, and L. Yang, "On optimal power allocation for downlink non-orthogonal multiple access systems," IEEE J. Sel. Areas Commun., vol. 35, no. 12, pp. 2744-2757, Dec. 2017.

[33] Z. Ding, Z. Yang, P. Fan, and H. V. Poor, "On the performance of non-orthogonal multiple access in $5 \mathrm{G}$ systems with randomly deployed users," IEEE Signal Process. Lett., vol. 21, no. 12, pp. 1501-1505, Dec. 2014.

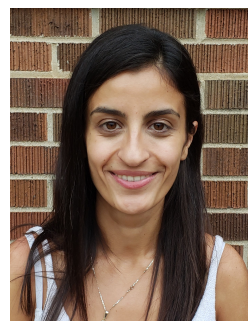

Marie-Josepha Youssef received her B.E. and M.S. degrees in computer and communications engineering in 2016 from the Lebanese University, Lebanon. She is currently working towards the Ph.D. degree in information and communication engineering at IMT Atlantique, France. Her current research interests include resource allocation, non-orthogonal multiple access, matching theory, reinforcement learning, unmanned-aerial vehicles and grant-free communications.

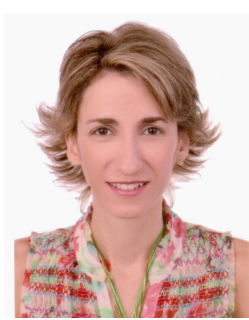

Joumana Farah received the B.E. degree in Electrical Engineering from the Lebanese University, in 1998, the M.E. degree in Signal, Image, and Speech processing, in 1999, and the Ph.D. degree in mobile communication systems, in 2002, from the University of Grenoble, France. In 2010, she received the Habilitation to Direct Research (HDR) from University Pierre and Marie Curie (Paris VI), France. She is currently a full-time professor at the Faculty of Engineering, Lebanese University, Lebanon. She has supervised a large number of Master, PhD theses and post-docs. She has received several research grants from the Lebanese National Council for Scientific Research, the FrancoLebanese CEDRE program, and the Lebanese University. She has nine registered patents and a software and has coauthored a research book and more than a hundred of papers in international journals and conferences. Her current research interests include resource allocation techniques, channel coding, channel estimation, and interference management techniques. She was the General Chair of the 19th International Conference on Telecommunications (ICT 2012), and serves as a TPC member and a reviewer for several journals and conferences. 


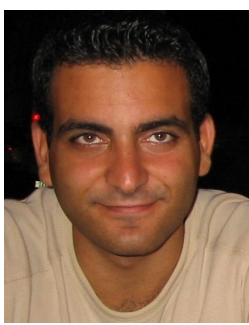

Charbel Abdel Nour obtained a computer and communications engineering degree in 2002 from the Lebanese University, a Master degree in digital communications from the University of Valenciennes, France, in 2003 and a Ph.D. in digital communications from IMT Atlantique, France in 2008. In June 2008 he joined the Electronics Department of IMT Atlantique as a post-doctoral fellow. His interests concern the radio mobile communications systems, broadcasting systems, coded modulation, error-correcting codes, MIMO and iterative receivers. Charbel has been involved in several research projects related to broadcasting, satellite and mobile communications. Since 2007, he has been active in the Digital Video Broadcasting DVB consortium where he had important contributions. Since November 2011, he holds an associate professor position at the Electronics Department of IMT Atlantique. With the increasing interest in next generation telecommunications systems, he participated to METIS, the largest project of the EU commission on future $5 \mathrm{G}$ systems on receiver and proof of concept aspects. He also participated to the ongoing 5GPPP FANTASTIC-5G project on aspects related to resource allocation, waveform and FEC design for 5G. Charbel provided several contributions to the 3GPP standardization body related to the FEC design. $\mathrm{He}$ is currently active in the Beyond 5G EU EPIC project related to ultrahigh throughput codes and decoders.

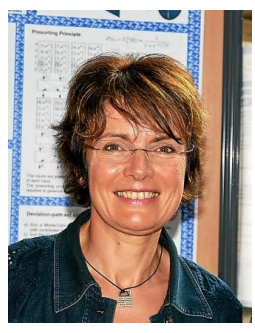

Catherine Douillard received the engineering degree in telecommunications from the Ecole Nationale Superieure des Telecommunications de Bretagne, France, in 1988, the Ph.D. degree in electrical engineering from the University of Western Brittany, Brest, France, in 1992, and the accreditation to supervise research from the University of Southern Brittany, Lorient, France, in 2004. She is currently a full Professor in the Electronics Department of Telecom Bretagne where she is in charge of the Algorithm-Silicon Interaction research team. Her main research interests are turbo codes and iterative decoding, iterative detection, the efficient combination of high spectral efficiency modulation and turbo coding schemes, diversity techniques and turbo processing for multicarrier, multi-antenna and multiple access transmission systems. In 2009, she received the SEE/IEEE Glavieux Award for her contribution to standards and related industrial impact. She was active in the DVB (Digital Video Broadcasting) Technical Modules for the definition of DVB-T2, DVB-NGH as chairperson of the "Coding, Constellations and Interleaving" task force and DVB-RCS NG standards. Since 2015, she has had several contributions in the FANTASTIC-5G and EPIC H2020 European projects intended for the definition of new techniques for $5 \mathrm{G}$ and beyond. 